Simplified techniques for predicting

the transmission loss of a circular dissipative silencer

\title{
Ray Kirby
}

Department of Mechanical Engineering,

Brunel University, Uxbridge, Middlesex, UB8 3PH, UK. 


\begin{abstract}
A closed-form analytical solution for the transmission loss of a dissipative silencer with a circular cross-section is described. The silencer contains a bulk reacting acoustic absorbent which is separated from a mean gas flow by a perforated screen. Theoretical predictions of the silencer transmission loss for three different dissipative silencers are compared both with experimental data and with another more complex modelling technique. Good agreement is noted between the analytical theory and experimental data in the low-to-medium frequency range. Below a defined upper frequency limit the analytical technique is also shown to provide good agreement with the finite element method. In addition it is observed that, even for relatively high open area porosities, the perforate screen has a significant effect on dissipative silencer performance.
\end{abstract}




\section{INTRODUCTION}

Dissipative silencers are commonly deployed to attenuate broadband noise emanating from internal combustion engines. In recent years, silencer modelling techniques have advanced considerably and methods are now available for studying automotive dissipative silencers of any shape or size. The advancement in design techniques has been accompanied by an increase in model complexity and the use of numerical formulations is now widespread. For example both the finite element method [1,2] and the boundary element method [3] have been used successfully to model dissipative silencers, although the finite element method is currently the most popular technique. Dissipative silencers of arbitrary shape, or even those with an arbitrary but uniform cross-section, require numerical techniques in order to obtain sufficiently accurate predictions [4]. Numerical methods are not, however, always required when studying simpler silencer geometries and opportunities exist for economising on model complexity and subsequent CPU run time, whilst retaining prediction accuracy.

Simple silencer geometries, such as a circular cross-section, are often found in automotive applications. The dissipative silencer typically consists of an expansion chamber, lined with a porous acoustically absorbent material surrounding a central airway in which the exhaust gas emanating from the engine is transported. To prevent loss of, or damage to the porous material, a concentric perforate screen typically separates the absorbent from the mean gas flow. The acoustic modelling of circular dissipative silencers is well established due to the relative simplicity of the geometry, although most studies concentrate on predicting modal

attenuation rates for infinite silencers. For example, Ko [5] derived a governing eigenequation for annular and circular ducts containing a bulk reacting liner and mean gas flow. Later, Nilsson and Brander [6] studied the effects of a perforate screen on modal 
attenuation rates in a lined infinite duct. The effect on modal attenuation rates of an internal mean flow within the bulk reacting liner was examined by Cummings and Chang [7], who solved the governing eigenequation for a circular silencer by using an iterative NewtonRaphson scheme. Frommhold and Mechel [8] chose to avoid iterative schemes and developed an analytic closed-form solution for attenuation rates in a dissipative silencer although the effects of mean flow and a perforate were omitted (see also reference [9]).

The prediction of modal attenuation rates in infinite silencers provides only a guide to overall silencer performance. To characterise more fully the acoustic performance of a finite length silencer it is also necessary to model the influence of the entry and exit planes of the silencer. This can readily be achieved provided modal attenuation rates have already been computed. For example Nilsson and Brander [10] employed the Wiener Hopf method to study the influence of the entry and exit planes in a dissipative silencer, after first calculating modal attenuation rates for a lined infinite duct [6]. A more straightforward technique, known as mode matching, was later utilised by Cummings and Chang [11] in order to calculate the transmission loss of a finite length silencer after first extracting six different modes from the governing eigenequation. Cummings and Chang matched axial acoustic particle velocity and acoustic pressure across the silencer discontinuities and good agreement between prediction and measurement was observed. Later, Peat [12] matched the average acoustic pressure and volume velocity across the silencer discontinuities and derived an explicit closed-form solution of the governing eigenequation before predicting the transmission loss of a dissipative silencer. The method of Peat [12] relied upon substituting low argument approximations for the Bessel and Neumann functions present in both the governing eigenequation and the matching conditions. This avoided iterative solution schemes but restricted transmission loss predictions to those calculated using the least attenuated mode 
only. Nevertheless, Peat [12] found good agreement with the multi-mode transmission loss predictions of Cummings and Chang [11] and proposed that discrepancies between the two methods at higher frequencies were caused only by the omission of higher order modes.

Explicit, closed form, algorithms capable of predicting modal attenuation rates and/or silencer transmission loss are attractive since they offer the potential for fast computation and avoid the problems associated with iterative schemes, such as sensitivity of the final solution to the initial guess and the "jumping" of modal solutions. Obviously, the benefits gained by using a simplified algorithm to predict silencer performance must be balanced against the desired prediction accuracy; nevertheless, the potential advantages of an analytical formulation make the investigation of such a technique worthwhile. In this paper, a simplified analytical approach to modelling circular dissipative silencers is described. An approach similar to the one adopted by Peat [12] is proposed here, but extra terms in the series expansion of the Bessel functions are included. The reasoning behind this is that the model presented by Peat is accurate only for relatively small silencers or at low frequencies, whereas the introduction of extra terms in the series expansions potentially allows larger dissipative silencers and/or higher frequencies to be modelled accurately.

The analysis adopted here splits conveniently into two parts. First the governing eigenequation for a circular dissipative silencer is solved by substituting series expansions for the Bessel and Neumann functions present in the eigenequation. The method follows the one described by Peat [12], but retains a greater number of terms in the series expansions, and a perforate screen is also introduced into the model. Secondly, transmission loss predictions are obtained by applying the mode matching technique described by Cummings and Chang [11], but retaining the fundamental modes only. Moreover the matching conditions at the 
silencer discontinuities are simplified by substituting series approximations for the Bessel and Neumann functions. The transmission loss predictions obtained are then compared against experimental measurement and also against predictions obtained by using the finite element method (see reference [1]). In order to provide a stringent test of the new method, larger silencers than those studied previously $[11,12]$ are chosen here and two different porous materials are examined.

\section{GOVERNING EQUATIONS}

The dissipative silencer studied in this section is assumed to have a uniform circular crosssection and to contain a uniform mean gas flow of Mach number $M$ in the central channel (see Figure 1). In the silencer chamber, a perforate screen separates the central channel (region 2) from the (isotropic) porous absorbent (region 3). The inlet and outlet pipes are denoted by regions 1 and 4 respectively. The theory presented here is based on previous work [7, 12], although the derivation is modified slightly to include the influence of the perforate screen and the omission of mean flow in the absorbent (negligible due to the presence of a perforate). To begin with coupled modal solutions are sought for the sound field in both the airway and the absorbent, with a common axial wavenumber linking the two regions, although the analysis is restricted to the fundamental, or least attenuated, mode only. 


\subsection{EIGENVALUE FORMULATION FOR AN AXISYMMETRIC DISSIPATIVE} SILENCER

The acoustic wave equation in region (2) is given by [7]

$$
\frac{1}{c_{0}^{2}} \frac{\mathrm{D}^{2} p_{2}^{\prime}}{\mathrm{D} t^{2}}-\nabla^{2} p_{2}^{\prime}=0
$$

where $c_{0}$ is the isentropic speed of sound, $p^{\prime}$ is the acoustic pressure and $t$ is time. Assuming a time dependence of $\mathrm{e}^{\mathrm{i} \omega t}$ (where $\mathrm{i}=\sqrt{-1}$ and $\omega$ is the radian frequency), equation (1) may be re-written as

$$
k_{0}^{2} p_{2}^{\prime}-2 \mathrm{i} M k_{0} \frac{\partial p_{2}^{\prime}}{\partial x}+\left(1-M^{2}\right) \frac{\partial^{2} p_{2}^{\prime}}{\partial x^{2}}+\frac{1}{r} \frac{\partial p_{2}^{\prime}}{\partial r}+\frac{\partial^{2} p_{2}^{\prime}}{\partial r^{2}}=0,
$$

where $k_{0}\left(=\omega / c_{0}\right)$ is the wavenumber and $M$ is the mean flow Mach number in the central airway. The acoustic wave equation in region 3 may be written [7] as

$$
\frac{\partial^{2} p_{3}^{\prime}}{\partial x^{2}}+\frac{1}{r} \frac{\partial p_{3}^{\prime}}{\partial r}+\frac{\partial^{2} p_{3}^{\prime}}{\partial r^{2}}-\Gamma^{2} p_{3}^{\prime}=0
$$

provided the porous medium is assumed to be isotropic with a propagation constant $\Gamma$. The coupled sound fields in regions (2) and (3) are now expressed as a function of the least attenuated incident and reflected propagating mode, i.e., 


$$
p_{c}^{\prime}(x, r)=P_{c_{i}} \Psi_{c_{i}}(r) \mathrm{e}^{-\mathrm{i} k_{0} k_{x_{i}} x}+P_{c_{r}} \Psi_{c_{r}}(r) \mathrm{e}^{-\mathrm{i} k_{0} k_{x_{r}} x}
$$

where $p_{c}^{\prime}(x, r)$ denotes the acoustic pressure in the chamber (regions (2) and (3) combined), $P_{c}$ is the modal coefficient, $\Psi_{c}(r)$ is the transverse modal eigenfunction, $k_{x}$ is the (coupled) axial wavenumber and $i$ refers to an incident wave, $r$ to a reflected wave. By substituting the assumed sound field in the chamber back into equations (2) and (3) and by taking a solution involving the positive travelling mode only, the acoustic pressure in regions 2 and 3 may be expressed as

$$
p_{2}^{\prime}(x, r)=P_{2_{i}} \mathrm{~J}_{0}\left(\alpha_{i} r\right) \mathrm{e}^{-\mathrm{i} k_{0} k_{x_{i}} x} \text { and } p_{3}^{\prime}(x, r)=P_{3_{i}}\left\{\mathrm{~J}_{0}\left(\beta_{i} r\right)-\frac{\mathrm{Y}_{0}\left(\beta_{i} r\right) \mathrm{J}_{1}\left(\beta_{i} r_{2}\right)}{\mathrm{Y}_{1}\left(\beta_{i} r_{2}\right)}\right\} \mathrm{e}^{-\mathrm{i} k_{0} k_{x_{i}} x}
$$

respectively, provided $p_{2}^{\prime}$ is finite at $r=0$ and we take $\partial p_{3}^{\prime} / \partial r=0$ at $r=r_{2}$. Here $\mathrm{J}_{\mathrm{m}}(\cdot \cdot)$ and $\mathrm{Y}_{\mathrm{m}}(\cdot)$ are $m^{\text {th }}$ order Bessel and Neumann functions, respectively, and the radial wavenumbers $\alpha_{i}$ and $\beta_{i}$ are given by

$$
\alpha_{i}^{2}=k_{0}^{2}\left[1-2 M k_{x_{i}}-\left(1-M^{2}\right) k_{x_{i}}^{2}\right] \text { and } \beta_{i}^{2}=-k_{0}^{2}\left[\tilde{\Gamma}^{2}+k_{x_{i}}^{2}\right]
$$

respectively, where $\tilde{\Gamma}=\Gamma / k_{0}$.

The governing eigenequation for the chamber is derived by applying continuity of normal particle displacement across the perforate screen and, at $r=r_{1}$, by enforcing

$$
p_{2}^{\prime}\left(x, r_{1}\right)-p_{3}^{\prime}\left(x, r_{1}\right)=\mathrm{i} \omega \rho_{0} c_{0} \zeta \xi_{2}^{\prime}\left(x, r_{1}\right)
$$


where $\zeta$ is the (dimensionless) acoustic impedance of the perforate and $\xi_{2}^{\prime}$ is the radial acoustic particle displacement in region 2. The assumption of an infinitesimally thin perforate is implicit in the application of the boundary conditions and is valid because the thickness of a perforate screen is typically small when compared to the overall silencer dimensions. Continuity of normal particle displacement at $r=r_{1}$ gives

$$
\xi_{2}^{\prime}\left(x, r_{1}\right)=\xi_{3}^{\prime}\left(x, r_{1}\right)
$$

where $\xi_{3}^{\prime}$ is the radial acoustic particle displacement in region 3. The linearised Euler equation gives the acoustic particle displacement in regions 2 and 3 as

$$
\xi_{2}^{\prime}=\left[1 / \rho_{0} \omega^{2}\left(1-M k_{x_{i}}\right)^{2}\right] \partial p_{2}^{\prime} / \partial r, \text { and } \xi_{3}^{\prime}=\left(1 / \rho(\omega) \omega^{2}\right) \partial p_{3}^{\prime} / \partial r
$$

respectively, where $\rho(\omega)$ is the equivalent bulk complex density of the porous material. Substitution of equations (9a) and (9b) into equations (7) and (8) links together the pressure fields in regions 2 and 3 and, after application of equations (5a) and (5b), an eigenequation for the chamber may be written as

$$
\begin{aligned}
& \left(\beta_{i} / \alpha_{i}\right)\left[\mathrm{J}_{1}\left(\beta_{i} r_{1}\right) \mathrm{Y}_{1}\left(\beta_{i} r_{2}\right)-\mathrm{J}_{1}\left(\beta_{i} r_{2}\right) \mathrm{Y}_{1}\left(\beta_{i} r_{1}\right)\right]\left(\left[1-M k_{x_{i}}\right]^{2} \mathrm{~J}_{0}\left(\alpha_{i} r_{1}\right) / \mathrm{J}_{1}\left(\alpha_{i} r_{1}\right)+\mathrm{i} \zeta \alpha_{i} / k_{0}\right) \\
& -\left(\rho(\omega) / \rho_{0}\right)\left[\mathrm{J}_{0}\left(\beta_{i} r_{1}\right) \mathrm{Y}_{1}\left(\beta_{i} r_{2}\right)-\mathrm{Y}_{0}\left(\beta_{i} r_{1}\right) \mathrm{J}_{1}\left(\beta_{i} r_{2}\right)\right]=0 .
\end{aligned}
$$

Equation (10) is identical to the governing eigenequation derived by Cummings and Chang [7] except for the introduction of an extra term accounting for the perforate screen and the 
assumption of an isotropic porous material. Furthermore, the transverse modal eigenfunctions are now given as

$$
\Psi_{i}(r)=\left\{\begin{array}{cc}
\Phi_{0}\left(\alpha_{i} r\right) & 0 \leq r \leq r_{1} \\
\mathrm{~J}_{0}\left(\beta_{i} r\right)-\mathrm{Y}_{0}\left(\beta_{i} r\right) \mathrm{J}_{1}\left(\beta_{i} r_{2}\right) / \mathrm{Y}_{1}\left(\beta_{i} r_{2}\right) & r_{1} \leq r \leq r_{2}
\end{array}\right\}
$$

where $\Phi=\left[\mathrm{J}_{0}\left(\beta_{i} r_{1}\right)-\frac{\mathrm{Y}_{0}\left(\beta_{i} r_{1}\right) \mathrm{J}_{1}\left(\beta_{i} r_{2}\right)}{\mathrm{Y}_{1}\left(\beta_{i} r_{2}\right)}\right] /\left[\mathrm{J}_{0}\left(\alpha_{i} r_{1}\right)+\mathrm{i} \frac{\zeta \alpha_{i} \mathrm{~J}_{1}\left(\alpha_{i} r_{1}\right)}{k_{0}\left(1-M k_{x_{i}}\right)^{2}}\right]$.

The eigenequation may be solved for the positive propagating axial wavenumber $k_{x_{i}}$ once the radial wavenumbers $\alpha_{i}$ and $\beta_{i}$ have been expressed in terms of the axial wavenumber by applying equations (7a) and (7b). Cummings and Chang [11] adopted an iterative NewtonRaphson scheme to solve the governing eigenequation and successfully calculated the axial wavenumber for six different eigenmodes. This method relies upon choosing a suitable initial guess for each individual mode and for dissipative silencers in general the method cannot be guaranteed to be free from phenomena such as modal "jumping" or other convergence problems. An alternative approach, adopted by Peat [12], is to solve the eigenequation analytically by substituting a series expansion for each Bessel and Neumann function present in the eigenequation. This method has the advantage of avoiding convergence problems although the accuracy of the predicted wavenumber depends upon the how many terms in the series expansion are retained. The latter approach is adopted in section 3 . 


\subsection{PREDICTION OF SILENCER TRANSMISSION LOSS}

The silencer transmission loss may be computed, once the axial wavenumber has been calculated, by matching the sound fields at each silencer discontinuity (see reference [11]). The approach of Cummings and Chang [11] is adopted here although their analysis is restricted to include only the least attenuated or fundamental mode. The mode matching scheme applies continuity of acoustic pressure and continuity of axial particle velocity across silencer planes A and B (see Figure 1). The assumption of plane wave propagation allows the sound pressure in regions 1 and 4 to be expressed as

$$
\begin{gathered}
p_{1}^{\prime}(x, r)=P_{1_{i}} \mathrm{e}^{-\mathrm{i} k_{0} x /(1+M)}+P_{1_{r}} \mathrm{e}^{\mathrm{i} k_{0} x /(1-M)}, \\
p_{4}^{\prime}(x, r)=P_{4_{i}} \mathrm{e}^{-\mathrm{i} k_{0} x^{\prime} /(1+M)},
\end{gathered}
$$

provided region 4 is anechoically terminated downstream of the silencer so that $P_{4_{r}}=0$. Applying continuity of acoustic pressure over planes A and B and integrating over the area of each plane [11] gives

$$
\begin{aligned}
& \int_{S_{1}} p_{1}^{\prime}(x, r) d S_{1}=\int_{S_{1}} p_{c}^{\prime}(x, r) d S_{1} \text { over plane A, } \\
& \int_{S_{1}} p_{c}^{\prime}(x, r) d S_{1}=\int_{S_{1}} p_{4}^{\prime}(x, r) d S_{1} \text { over plane B, }
\end{aligned}
$$

where $S_{1}$ is the area of the airway. Thus by substituting equations (4), (12) and (13) into the pressure matching conditions we may write 


$$
\pi r_{1}^{2}\left[P_{1_{i}}+P_{1_{r}}\right]=P_{c_{i}} \int_{S_{1}} \Psi_{c_{i}} d S_{1}+P_{c_{r}} \int_{S_{1}} \Psi_{c_{r}} d S_{1},
$$

at $x=0$ and

$$
\pi r_{1}^{2} P_{4_{i}}=P_{c_{i}} \mathrm{e}^{-\mathrm{i} k_{0} k_{x_{i}} L} \int_{S_{1}} \Psi_{c_{i}} d S_{1}+P_{c_{r}} \mathrm{e}^{-\mathrm{i} k_{0} k_{x_{r}} L} \int_{S_{1}} \Psi_{c_{r}} d S_{1}
$$

at $x^{\prime}=0$, where $L$ is the length of the silencer chamber.

Similarly, continuity of volume velocity over planes A and B gives

$$
\begin{aligned}
& \int_{S_{1}} u_{x_{1}}^{\prime}(x, r) d S_{1}=\int_{S_{c}} u_{x_{c}}^{\prime}(x, r) d S_{c} \text { over plane A, } \\
& \int_{S_{c}} u_{x_{c}}^{\prime}(x, r) d S_{c}=\int_{S_{1}} u_{x_{4}}^{\prime}(x, r) d S_{1} \text { over plane } \mathrm{B},
\end{aligned}
$$

where $u_{x}^{\prime}$ denotes axial acoustic particle velocity. After application of the linearised Euler equation the velocity matching conditions may be written as

$$
\pi r_{1}^{2}\left[P_{1_{i}}-P_{1_{r}}\right]=P_{c_{i}} A_{i} \int_{S_{1}} \Psi_{c_{i}} d S_{1}+P_{c_{r}} A_{r} \int_{S_{1}} \Psi_{c_{r}} d S_{1}
$$

at $x=0$ and

$$
\pi r_{1}^{2} P_{4_{i}}=P_{c_{i}} A_{i} \mathrm{e}^{-\mathrm{i} k_{0} k_{x_{i}} L} \int_{S_{1}} \Psi_{c_{i}} d S_{1}+P_{c_{r}} A_{r} \mathrm{e}^{-\mathrm{i} k_{0} k_{x_{r}} L} \int_{S_{1}} \Psi_{c_{r}} d S_{1}
$$

at $x^{\prime}=0$, where

$$
A_{i, r}=\left\{\begin{array}{ll}
k_{x_{i, r}} /\left(1-M k_{x_{i, r}}\right) & 0 \leq r \leq r_{1} \\
\rho_{0} k_{x_{i, r}} / \rho(\omega) & r_{1} \leq r \leq r_{2}
\end{array}\right\} .
$$


Equations (16), (17), (20) and (21) may now be solved for unknown modal coefficients $P_{1_{r}}$, $P_{c_{i}}, P_{c_{r}}$ and $P_{4_{i}}$ after setting $P_{1_{i}}$ equal to unity (real). The sound transmission loss of the silencer is then given by [11]

$$
T L=-20 \log \left|P_{4_{i}}\right|
$$

The prediction of the silencer transmission loss is straightforward, once the integrals in equations (16), (17), (20) and (21) have been computed. Cummings and Chang [11] employed numerical methods to compute these integrals, however for a circular silencer it is possible perform each integration analytically. Moreover, the eigenfunctions in the chamber are products only of Bessel and Neumann functions and so series expansions can be substituted into each expression in much the same way as for the governing eigenequation. This procedure will enable an explicit algorithm to be written for predicting the silencer transmission loss and the development of this will be discussed in the next section.

\section{SIMPLIFIED TECHNIQUES FOR CALCULATING SILENCER TRANSMISSION}

\section{LOSS}

In this section an analytical technique is proposed for calculating the transmission loss of a circular dissipative silencer. To begin with the eigenequation derived in section 2.1 (equation (10)) is re-written by substituting series expansions for the Bessel and Neumann functions, neglecting terms of $O\left(\alpha_{i}^{5} r_{1}^{5}\right), O\left(\beta_{i}^{5} r_{2}^{5}\right)$ and above. This approximation implies "low" values of $\alpha_{i}, \beta_{i}$ and/or a relatively small silencer. However, it is common, for a fixed silencer 
geometry, to define an upper frequency limit, beyond which the approximation is no longer valid. Consequently such an approach is often termed a low frequency approximation and this terminology will be adopted here.

\subsection{LOW FREQUENCY APPROXIMATION OF GOVERNING EIGENEQUATION}

Employing a series expansion for each Bessel and Neumann function (see reference [13]) in equation (10) and neglecting terms of $O\left(\alpha_{i}^{5} r_{1}^{5}\right)$ and $O\left(\beta_{i}^{5} r_{2}^{5}\right)$ allows the eigenequation to be re-written as

$$
\begin{aligned}
\frac{\beta_{i}^{2}}{\alpha_{i}^{2}}\{[1- & \left.M k_{x_{i}}\right]^{2}\left(1-\frac{\alpha_{i}^{2} r_{1}^{2}}{4}+\frac{\beta_{i}^{2} r_{2}^{2} R_{2}}{8}-\frac{\alpha_{i}^{2} r_{1}^{2} \beta_{i}^{2} r_{2}^{2} R_{2}}{32}+\frac{\alpha_{i}^{4} r_{1}^{4}}{64}-\frac{\beta_{i}^{4} r_{2}^{4} R_{4}}{192}\right) \\
+ & \left.\frac{\mathrm{i} \zeta \alpha_{i}^{2} r_{1}}{2 k_{0}}\left(1-\frac{\alpha_{i}^{2} r_{1}^{2}}{8}+\frac{\beta_{i}^{2} r_{2}^{2} R_{2}}{8}-\frac{\alpha_{i}^{2} r_{1}^{2} \beta_{i}^{2} r_{2}^{2} R_{2}}{64}+\frac{\alpha_{i}^{4} r_{1}^{4}}{192}-\frac{\beta_{i}^{4} r_{2}^{4} R_{4}}{192}\right)\right\} \\
+ & \frac{S_{1}}{S_{2}} \frac{\rho(\omega)}{\rho_{0}}\left(1-\frac{\alpha_{i}^{2} r_{1}^{2}}{8}-\frac{\beta_{i}^{2} r_{2}^{2} R_{1}}{4}+\frac{\alpha_{i}^{2} r_{1}^{2} \beta_{i}^{2} r_{2}^{2} R_{1}}{32}+\frac{\alpha_{i}^{4} r_{1}^{4}}{192}+\frac{\beta_{i}^{4} r_{2}^{4} R_{3}}{64}\right)=0,
\end{aligned}
$$

where $S_{1}=\pi r_{1}^{2}, S_{2}=\pi\left(r_{2}^{2}-r_{1}^{2}\right)$ and $R_{1} \ldots R_{4}$ are dimensionless functions of the silencer geometry given by

$$
\begin{gathered}
R_{1}=\frac{r_{1}^{2}}{r_{2}^{2}}-1+2 \ln \left(\frac{r_{2}}{r_{1}}\right), \quad R_{2}=\frac{S_{1}}{S_{2}}\left[\frac{r_{1}^{2}}{r_{2}^{2}}-\frac{r_{2}^{2}}{r_{1}^{2}}+4 \ln \left(\frac{r_{2}}{r_{1}}\right)\right] \quad(25 \mathrm{a}, \mathrm{b}) \\
R_{3}=\frac{r_{1}^{4}}{r_{2}^{4}}-5+4 \frac{r_{1}^{2}}{r_{2}^{2}}+4\left(2 \frac{r_{1}^{2}}{r_{2}^{2}}+1\right) \ln \left(\frac{r_{2}}{r_{1}}\right), \quad R_{4}=\frac{S_{1}}{S_{2}}\left[\frac{r_{1}^{4}}{r_{2}^{4}}-\frac{r_{2}^{2}}{r_{1}^{2}}+9\left(\frac{r_{1}^{2}}{r_{2}^{2}}-1\right)+12\left(\frac{r_{1}^{2}}{r_{2}^{2}}+1\right) \ln \left(\frac{r_{2}}{r_{1}}\right)\right] .
\end{gathered}
$$


Retaining terms up to and including $O\left(\alpha_{i}^{4} r_{1}^{4}\right)$ and $O\left(\beta_{i}^{4} r_{2}^{4}\right)$ may seem excessive in comparison to the method used by Peat [12], who obtained satisfactory transmission loss predictions after neglecting terms of $O\left(\alpha_{i}^{2} r_{1}^{2}\right)$ and $O\left(\beta_{i}^{2} r_{2}^{2}\right)$. Moreover, Peat proposed that the small discrepancies between his transmission loss predictions and the multi-mode predictions of Cummings and Chang [11] at higher frequencies, were caused by the omission of higher order modes and not by neglecting higher order terms in the series expansions of the Bessel and Neumann functions. However, if one examines larger silencers, such as those studied later on in this paper, the series expansions used by Peat [12] are no longer sufficiently accurate when predicting the axial wavenumber over the frequency range of interest (up to approximately $1.5 \mathrm{kHz}$ ). It appears that neglecting terms of $O\left(\alpha_{i}^{2} r_{1}^{2}\right)$, $O\left(\beta_{i}^{2} r_{2}^{2}\right)$ and above provides the underlying cause of the discrepancies between Peat's transmission loss calculations and those of Cummings and Chang [11], not the omission of higher order modes. Therefore, to maintain accurate predictions when examining larger silencers it is necessary, as one would expect, to retain a greater number of terms in the series expansions of the Bessel and Neumann functions, hence the retention of terms up to and including $O\left(\alpha_{i}^{4} r_{1}^{4}\right)$ and $O\left(\beta_{i}^{4} r_{2}^{4}\right)$ in the governing eigenequation. This issue is, however, complicated further when the expressions for the radial wavenumbers (equations (6a) and (6b)) are substituted into equation (24), allowing the eigenequation to be written explicitly in terms of $k_{x_{i}}$. The dimensionless propagation constant $\tilde{\Gamma}$ appears (as $\tilde{\Gamma}^{2}$ ) in equation (6b) and, for typical automotive silencers covering a frequency range up to approximately $1.5 \mathrm{kHz}$, the value of $\tilde{\Gamma}^{2}$ is large when compared to $k_{0}^{2} r_{1}^{2}$ and $k_{0}^{2} r_{2}^{2}$. For example if one examines the measurements reported by Delany and Bazley [14] for a range of fibrous porous materials (fibrous porous materials such as glass fibre and rock wool are common in automotive silencers), it is evident that for these materials, which have relatively high flow resistivities, 
values for $\tilde{\Gamma}^{2}$ are large at low frequencies when compared to $k_{0}^{2} r_{1}^{2}$ and $k_{0}^{2} r_{2}^{2}$. Therefore, terms in series expansions which contain $\tilde{\Gamma}^{2}$ are not of the same order as equivalent terms which do not contain $\tilde{\Gamma}^{2}$. For the silencers studied here, terms of $O\left(k_{0}^{2} r_{1}^{2}\right)$ and $O\left(k_{0}^{2} r_{2}^{2}\right)$ are in fact similar to those terms of $O\left(k_{0}^{4} r_{1}^{4} \tilde{\Gamma}^{2}\right)$ and $O\left(k_{0}^{4} r_{2}^{4} \tilde{\Gamma}^{2}\right)$, terms of $O(1)$ are similar to $O\left(k_{0}^{2} r_{1}^{2} \tilde{\Gamma}^{2}\right)$ and $O\left(k_{0}^{2} r_{2}^{2} \tilde{\Gamma}^{2}\right)$, and so on. In the analysis which follows, the governing eigenequation is written explicitly as a function of $k_{x_{i}}$ and only terms up to and including $O\left(k_{0}^{2} r_{1}^{2}\right)$ and $O\left(k_{0}^{2} r_{2}^{2}\right)$ are retained. This does however mean that terms of $O\left(k_{0}^{4} r_{1}^{4} \tilde{\Gamma}^{2}\right)$ and $O\left(k_{0}^{4} r_{2}^{4} \tilde{\Gamma}^{2}\right)$ must also be included and this is the reason behind retaining terms up to and including $O\left(\alpha_{i}^{4} r_{1}^{4}\right)$ and $O\left(\beta_{i}^{4} r_{2}^{4}\right)$ in equation (24).

After substituting expressions for the radial wavenumbers $\alpha_{i}$ and $\beta_{i}$ into equation (24) and neglecting terms of $O\left(k_{0}^{3} r_{1}^{3}\right), O\left(k_{0}^{3} r_{2}^{3}\right)$ and above, the governing eigenequation may be written as

$$
H_{00}+H_{11} M k_{x_{i}}+\left(H_{20}+H_{22} M^{2}\right) k_{x_{i}}^{2}-H_{31} M k_{x_{i}}^{3}-\left(H_{40}-H_{42} M^{2}\right) k_{x_{i}}^{4}+H_{51} M k_{x_{i}}^{5}-H_{62} M^{2} k_{x_{i}}^{6}=0 .
$$

The coefficients of equation (27) are listed in Appendix A. The solution of equation (27) proceeds by expanding $k_{x_{i}}$ in the form [12]

$$
k_{x_{i}}=a+b M+c M^{2}+\cdots,
$$

ignoring terms of $O\left(M^{3}\right)$ and above. Substituting equation (28) into equation (27) and equating terms of similar order in $M$ gives 


$$
\begin{gathered}
a= \pm\left\{\frac{H_{20} \pm \sqrt{H_{20}^{2}-4 H_{00} H_{40}}}{2 H_{40}}\right\}^{\frac{1}{2}}, \quad b=\frac{2 H_{11}-a^{2} H_{31}-a^{4} H_{51}}{4 a^{2} H_{40}-2 H_{20}}, \\
c=\frac{2 b H_{11}+b^{2} H_{20}-a^{2} H_{22}-3 a^{2} b H_{31}-6 a^{2} b^{2} H_{40}+a^{4} H_{42}-5 a^{4} b H_{51}+a^{6} H_{62}}{2 a\left[2 a^{2} H_{40}-H_{20}\right]} .
\end{gathered}
$$

The roots of the zero flow coefficient $a$, give rise to the incident and reflected waves, hence

$$
k_{x_{i}}=a+b M+c M^{2}, \quad k_{x_{r}}=-a+b M-c M^{2} .
$$

Note here that the roots inside the bracket of the zero flow coefficient give rise both to the least attenuated mode and the second least attenuated mode (the governing eigenequation (10) is in fact valid for higher order modes as well).

For a given frequency, the least attenuated axial wavenumber has been written explicitly in terms of the silencer geometry, absorbent material properties, perforate impedance and mean flow Mach Number. This has been achieved by using a series expansion for the Bessel and Neumann functions in the governing eigenequation and terminating these expansions at $O\left(k_{0}^{2} r_{1}^{2}\right)$ and $O\left(k_{0}^{2} r_{2}^{2}\right)$. Of course one is at liberty to choose where these series expansions should be terminated, however, the inclusion of terms of $O\left(k_{0}^{3} r_{1}^{3}\right), O\left(k_{0}^{3} r_{2}^{3}\right)$ and above precludes the writing of a general solution in the form shown above since a cubic or higher order equation appears for the zero flow coefficient. 


\subsection{LOW FREQUENCY ALGORITHM FOR SILENCER TRANSMISSION LOSS}

After calculating the incident and reflected axial wavenumbers for the least attenuated mode, the silencer transmission loss may be calculated using the matching technique described in section 2.2. By dividing the two pressure matching conditions (equations (16) and (17)) and the two velocity matching conditions (equations (20) and (21)) by the area in region (1) these may be re-written as

$$
\begin{gathered}
P_{1_{i}}+P_{1_{r}}=P_{c_{i}} I_{1_{i}}+P_{c_{r}} I_{1_{r}}, \\
P_{4_{i}}=P_{c_{i}} I_{1_{i}} \mathrm{e}^{-\mathrm{i} k_{0} k_{x_{i}} L}+P_{c_{r}} I_{1_{r}} \mathrm{e}^{-\mathrm{i} k_{0} k_{x_{r}} L}
\end{gathered}
$$

and

$$
\begin{gathered}
P_{1_{i}}-P_{1_{r}}=P_{c_{i}}\left[\frac{k_{x_{i}}}{\left(1-M k_{x_{i}}\right)} I_{1_{i}}+\frac{\rho_{0} k_{x_{i}}}{\rho(\omega)} I_{2_{i}}\right]+P_{c_{r}}\left[\frac{k_{x_{r}}}{\left(1-M k_{x_{r}}\right)} I_{1_{r}}+\frac{\rho_{0} k_{x_{r}}}{\rho(\omega)} I_{2_{r}}\right], \\
P_{4_{i}}=P_{c_{i}} \mathrm{e}^{-\mathrm{i} k_{0} k_{x_{i}} L}\left[\frac{k_{x_{i}}}{\left(1-M k_{x_{i}}\right)} I_{1_{i}}+\frac{\rho_{0} k_{x_{i}}}{\rho(\omega)} I_{2_{i}}\right]+P_{c_{r}} \mathrm{e}^{-\mathrm{i} k_{0} k_{x_{r}} L}\left[\frac{k_{x_{r}}}{\left(1-M k_{x_{r}}\right)} I_{1_{i}}+\frac{\rho_{0} k_{x_{r}}}{\rho(\omega)} I_{2_{r}}\right],
\end{gathered}
$$

where

$$
I_{1_{i, r}}=\frac{2}{r_{1}^{2}} \int_{0}^{r_{1}} \Psi_{i, r}(r) r d r \quad \text { and } \quad I_{2_{i, r}}=\frac{2}{r_{1}^{2}} \int_{r_{1}}^{r_{2}} \Psi_{i, r}(r) r d r
$$

Equations (31) to (34) may be solved for the modal coefficient $P_{4_{i}}$, by first setting $P_{1_{i}}=(1+\mathrm{i} 0)$ then by writing 


$$
P_{4_{i}}=\frac{2 I_{1_{i}}\left[\tilde{A}_{i}-\tilde{A}_{r}\right] \mathrm{e}^{-\mathrm{i} k_{0} k_{x_{i}} L}}{\left[I_{1_{i}}+\tilde{A}_{i}\right]\left[I_{1_{r}}-\tilde{A}_{r}\right]-\left[I_{1_{r}}+\tilde{A}_{r}\right]\left[I_{1_{i}}-\tilde{A}_{i}\right] \mathrm{e}^{-\mathrm{i} k_{0}\left(k_{x_{i}}-k_{x_{r}}\right) L}}
$$

where

$$
\tilde{A}_{i, r}=\frac{k_{x_{i, r}}}{\left(1-M k_{x_{i, r}}\right)} I_{1_{i, r}}+\frac{\rho_{0} k_{x_{i, r}}}{\rho(\omega)} I_{2_{i, r}} .
$$

The silencer transmission loss is calculated by using equation (23). Thus, the transmission loss of the silencer may be found once the integrals in equations (35) and (36) have been computed. The analytical computation of these integrals, and subsequent low argument approximation of the Bessel and Neumann functions, therefore completes the closed-form analytical solution for the silencer transmission loss. Hence by substituting equation (11) into equations (35) and (36), computing the integrals and applying low argument approximations for the Bessel and Neumann functions, one arrives at the following expressions:

$$
I_{1_{i, r}}=\left[\frac{1}{\Theta_{i, r}}\right]\left\{1-\frac{\alpha_{i, r}^{2} r_{1}^{2}}{8}-\frac{R_{1} \beta_{i, r}^{2} r_{2}^{2}}{4}-\frac{R_{1} \tilde{\Gamma}^{2} k_{0}^{2} \alpha_{i, r}^{2} r_{1}^{2} r_{2}^{2}}{32}+\frac{R_{3} \tilde{\Gamma}^{2} k_{0}^{4} r_{2}^{4}}{64}\left[\tilde{\Gamma}^{2}+2 k_{x_{i, r}}^{2}\right]\right\}
$$

where

$$
\begin{aligned}
\Theta_{i, r}= & -\frac{\alpha_{i, r}^{2} r_{1}^{2}}{4}\left(1-\frac{2 \mathrm{i} \zeta}{k_{0} r_{1}\left[1-M k_{x_{i, r}}^{2}\right]}\right)\left\{1+\frac{\tilde{\Gamma}^{2} k_{0}^{2} r_{2}^{2}}{2}\left[\ln \left(\frac{\beta_{i, r} r_{2}}{2}\right)+\tilde{\gamma}-0.5\right]\right\} \\
& -\frac{\beta_{i, r}^{2} r_{2}^{2}}{2}\left[\ln \left(\frac{\beta_{i, r} r_{2}}{2}\right)+\tilde{\gamma}-0.5\right]+\frac{\tilde{\Gamma}^{2} k_{0}^{4} r_{2}^{4}}{16}\left\{\tilde{\Gamma}^{2}+2 k_{x_{i, r}}^{2}\right\}\left[\ln \left(\frac{\beta_{i, r} r_{2}}{2}\right)+\tilde{\gamma}-1.25\right],
\end{aligned}
$$

and 


$$
I_{2_{i, r}}=\frac{\frac{S_{2}}{S_{1}}\left\{1+\frac{R_{2} \beta_{i, r}^{2} r_{2}^{2}}{8}-\frac{\left.R_{4} \tilde{\Gamma}^{2} k_{0}^{4} r_{2}^{4}\left[\tilde{\Gamma}^{2}+2 k_{x_{i, r}}^{2}\right]\right\}}{192}\left\{1-\frac{\beta_{i, r}^{2} r_{2}^{2}}{2}\left[\ln \left(\frac{\beta_{i, r} r_{2}}{2}\right)+\tilde{\gamma}-0.5\right]+\frac{\tilde{\Gamma}^{2} k_{0}^{4} r_{2}^{4}}{16}\left[\tilde{\Gamma}^{2}+2 k_{x_{i, r}}^{2}\right]\left[\ln \left(\frac{\beta_{i, r} r_{2}}{2}\right)+\tilde{\gamma}-1.25\right]\right\}\right.}{,}
$$

where $\tilde{\gamma}=0.577215665$ (Euler's Constant).

To arrive at equations (39) and (40), terms of $O\left(k_{0}^{3} r_{1}^{3}\right), O\left(k_{0}^{3} r_{2}^{3}\right)$ and above have been omitted, in accordance with the procedure adopted in Section 3.1. The silencer transmission loss has now been written explicitly in terms of the silencer geometry, absorbent material properties, perforate impedance and mean flow Mach number and may be computed for any given frequency.

\section{EXPERIMENT}

\subsection{SILENCER TRANSMISSION LOSS}

Experimental tests were performed on three, relatively large, circular silencers containing a bulk reacting porous material and a concentric perforate screen. Two different types of fibre glass, E glass and A glass, were used as the absorbent materials. The chamber dimensions for each silencer are given in Table 1 (for each silencer $r_{1}=37 \mathrm{~mm}$ ). A laboratory method convenient for measuring silencer transmission loss is the impulse technique described by Cummings and Chang [11]. This method is appropriate in the absence of an anechoic chamber and is also more suited to tests which involve mean flow. The experimental apparatus was arranged as shown in Figure 2. A rectangular pulse is delivered by the 
loudspeaker and measured by a microphone placed downstream of the silencer. To filter out unwanted flow noise, the signal was successively triggered by the output signal from the function generator, and averaged on the analyser up to 1024 times in the time domain. The averaged signal was then edited in the time domain to remove "spurious" data such as signal reflections from the pipe terminations. The measurements were then repeated but with the silencer removed from the test rig. To calculate the silencer transmission loss, the time domain signals captured with and without a silencer present are first transformed into the frequency domain by applying a discrete Fourier transform, the transmission loss is then calculated by taking the logarithmic ratio of the two spectra [11].

The effectiveness of the impulse technique for measuring silencer transmission loss depends heavily upon the pulse signal fed into the silencer and also the editing of the signal captured by the microphone. Ideally, over the frequency range of interest, the pressure amplitude of the pulse immediately upstream of the silencer should be constant. Although this criterion is easily initiated at the function generator, a combination of the amplifier, loudspeaker and side branch (see Figure 2) serve to distort the pressure amplitude of the signal before it reaches the silencer. In the tests performed here, a roll off in pressure amplitude was noticeable at the extremes of the frequency range of interest. Hence, in these frequency regions the signal to noise ratio may not be sufficiently large enough to provide sensible data. In addition, editing "spurious" data after capturing the signal at the microphone may remove small portions of data which are characteristic of the silencer itself. This is because internal reflections within the silencer may still be present by the time a spurious reflection from a termination arrives back at the microphone. The obvious remedy is to employ very long sections of pipe, either side of the silencer, and delay for as long as possible the arrival of unwanted reflections. However, laboratory dimensions restricted the size of the test rig to those shown in Figure 2 
and inevitably, when editing the microphone signal, a small portion of the signal characterising silencer performance was lost. Spurious reflections most notably affect transmission loss measurements taken in the low frequency range, since low frequency reflections within the dissipative silencer typically take the longest to die away. Thus, the overall effect of spurious reflections, combined with the roll off in pressure amplitude of the input signal, is to impose experimental frequency limits, outside of which the transmission loss measurements may be unreliable. For the test rig used here the transmission loss measurements are deemed to be reliable over a frequency range of approximately 150 to $1500 \mathrm{~Hz}$. To verify this frequency range a simple expansion chamber (identical to silencer A but with the absorbent material and perforate removed) was tested. Measurements were compared to theoretical predictions obtained using commercial design software (see Peat [15]) and agreement between the two was observed to be good (within 10\%) between 150 and $1750 \mathrm{~Hz}$ when no mean flow was present (see reference [4] for a more detailed discussion). Obviously, when mean flow is present experimental errors increase due to the effect of flow noise; however, a frequency range of approximately 150-1500 Hz appears reasonable for the apparatus used here.

\subsection{BULK ACOUSTIC PROPERTIES OF THE POROUS MATERIALS}

Fibre glass is commonly used as an acoustic absorbent in automotive silencers. Two different types of fibre glass were studied here, E glass, which has an average fibre diameter of approximately $5-13 \mu \mathrm{m}$, and A glass which has a larger average fibre diameter of approximately $18-26 \mu \mathrm{m}$. A popular technique for predicting the propagation constant and characteristic impedance of a bulk reacting porous material is the empirical power-law 
method described by Delany and Bazley [14]. This method is known, however, to produce non-physical predictions at low frequencies. To overcome this problem Kirby and Cummings [16] proposed a semi-empirical model which combines the empirical power-law method of Delany and Bazley with a theoretical microstructure model at low frequencies. Values for the propagation constant and characteristic impedance $\left(z_{a}\right)$ were given by Kirby and Cummings as

$$
\begin{aligned}
& \tilde{\Gamma}=\mathrm{i} \sqrt{\gamma_{0} q^{2}(\omega)}\left\{\frac{[\ln (1-\Omega)+1+2 \Omega] \Omega \ln (1-\Omega)+\Omega^{2}+3 \Omega^{2} / 2+\Omega^{3} / 3}{\left[\ln (1-\Omega)+\Omega+\Omega^{2} / 2\right]^{2}}-\left(\frac{\gamma_{0}-1}{\gamma_{0}}\right) \operatorname{Pr}-\mathrm{i} \frac{\Omega}{2 \pi \xi_{f} q_{0}^{2} s^{2}(\omega)}\right\}^{\frac{1}{2}}, \\
& \frac{z_{a}}{\rho_{0} c_{0}}=\sqrt{\frac{q^{2}(\omega)}{\gamma_{0} \Omega^{2}}}\left\{\frac{[\ln (1-\Omega)+1+2 \Omega] \Omega \ln (1-\Omega)+\Omega^{2}+3 \Omega^{2} / 2+\Omega^{3} / 3}{\left[\ln (1-\Omega)+\Omega+\Omega^{2} / 2\right]^{2}}+\left(\frac{\gamma_{0}-1}{\gamma_{0}}\right) \operatorname{Pr}-\mathrm{i} \frac{\Omega}{2 \pi \xi_{f} q_{0}^{2} s^{2}(\omega)}\right\}^{\frac{1}{2}},
\end{aligned}
$$

where $\Omega$ is the porosity of the porous material, $\xi_{f}$ is a dimensionless frequency parameter ( $\xi_{f}=\rho_{0} f / \sigma_{b}$, where $f$ is the frequency and $\sigma_{b}$ is the flow resistivity of the bulk porous material), $\gamma_{0}$ is the ratio of specific heats for air, Pr is the Prandtl number and the tortuosity $q^{2}(\omega)$ and shape factor $s^{2}(\omega)$ are given by

$$
\begin{aligned}
& q^{2}(\omega)=\frac{\left[\left(1+a_{3} \xi_{f}^{a_{4}}\right)\left(1+a_{5} \xi_{f}^{a_{6}}\right)-a_{1} a_{7} \xi_{f}^{\left(a_{2}+a_{8}\right)}\right]\left[\ln (1-\Omega)+\Omega+\Omega^{2} / 2\right]^{2}}{[\ln (1-\Omega)+1+2 \Omega] \ln (1-\Omega)+\Omega+3 \Omega^{2} / 2+\Omega^{3} / 3}, \\
& s^{2}(\omega)=\frac{q^{2}(\omega)}{2 \pi \xi_{f} q_{0}^{2}} \frac{1}{\left[a_{1} \xi_{f}^{a_{2}}\left(1+a_{5} \xi_{f}^{a_{6}}\right)+a_{7} \xi_{f}^{a_{8}}\left(1+a_{3} \xi_{f}^{a_{4}}\right)\right]},
\end{aligned}
$$


where $a_{1} \ldots a_{8}$ are Delany and Bazley coefficients measured experimentally, and $q_{0}^{2}$ is the socalled steady flow tortuosity. Values for the Delany and Bazley coefficients, the porosity of the material, the steady flow tortuosity and the steady flow resistivity (calculated for a material bulk density of $120 \mathrm{~kg} / \mathrm{m}^{3}$ ) are given for E glass and A glass in Table 2. Table 2 also lists a transition value for $\xi_{f}$, denoted here $\xi_{f_{0}}$, and this defines a value for $\xi_{f}$, below which $q^{2}(\omega)$ must be set equal to $q_{0}^{2}$ in equations (41) and (42), see reference [16].

\subsection{ACOUSTIC IMPEDANCE OF PERFORATE SCREEN}

Perforate screens are common in dissipative silencers and prevent loss of or damage to the porous material. The silencers studied here were constructed using a flat plate perforated with circular holes and formed into a concentric screen. The acoustic impedance of a perforate plate was shown by Kirby and Cummings [17] to increase when backed by a porous material. Kirby and Cummings formulated a semi-empirical model for the perforate impedance by adding a theoretical expression accounting for the effect of the porous material onto experimental data obtained for a perforate with no porous backing. The following relationships were proposed for the impedance of a plate perforated with circular holes, backed by a porous material and subjected to grazing gas flow [17],

$$
\zeta=\left\{\zeta^{\prime}-\mathrm{i} 0.425 k_{0} d+0.425 d z_{a} \Gamma / \rho_{0} c_{0}\right\} / \sigma
$$


where $d$ is the diameter of the hole, $\sigma$ is the area porosity of the perforate and $\zeta^{\prime}$ is the orifice impedance in the absence of a porous backing which may be written in terms of its resistive and reactive components, i.e.,

$$
\zeta^{\prime}=\theta+\mathrm{i} \chi
$$

Here, the orifice resistance $\theta$ is given by

$$
\theta=\left(26.16\left[\frac{t}{d}\right]^{-0.169}-20\right) \frac{u_{*}}{c_{0}}-0.6537 k_{0} d+\frac{t}{d} \sqrt{8 k_{0} v / c_{0}}
$$

where $t$ is the thickness of the plate, $v$ is the kinematic viscosity and $u_{*}$ is the friction velocity of the mean gas flow measured on the wall of the pipe. The orifice reactance $\chi$ is given by

$$
\chi=\mathrm{i} k_{0}(\delta+t),
$$

where

$$
\begin{array}{ll}
\frac{\delta}{\delta_{0}}=1, & u_{*} / f t \leq 0.18 d / t, \\
\frac{\delta}{\delta_{0}}=\left(1+0.6 \frac{t}{d}\right) \exp \left\{-\frac{u_{*} / f t-0.18 d / t}{1.8+t / d}\right\}-0.6 t / d, & u_{*} / f t>0.18 d / t,
\end{array}
$$

and $\delta_{0}=0.849 d$.

When no mean flow is present, the orifice resistance and orifice reactance are given by [18] 


$$
\theta=(1+t / d) \sqrt{8 k_{0} v / c_{0}}, \text { and } \chi=\mathrm{i} k_{0}(0.25 d+t)
$$

where $\theta$ and $\chi$ may be substituted into equation (46) in the same way as for the impedance values calculated when mean flow is present. The value for the orifice impedance with a porous backing is then calculated by using equation (45).

\section{RESULTS AND DISCUSSION}

In this section, transmission loss predictions obtained using the low frequency analytical technique described in section 3 are compared both with experimental measurement and with other theoretical modelling techniques. The primary aim here is to investigate how well the low frequency predictions compare with experimental measurements; however, a comparison is made also with predictions obtained using a more sophisticated modelling technique since this may provide further insight into the limitations of the model presented here.

Experimental measurements of silencer transmission loss were carried out for silencers A, B and $\mathrm{C}$ with mean flow Mach numbers of $M=0$ and 0.15 . Measurements were taken in the laboratory, using air as the working fluid. Results are reported in Figures 3-6 for silencer A (with $M=0$ and 0.15 ) and silencers $\mathrm{B}$ and $\mathrm{C}$ with $M=0.15$. The effect of flow noise is instantly recognisable in Figures 4-6 as rapid fluctuations in the transmission loss measurements, such fluctuations are inevitable when mean flow is present. An identical perforate screen is present in each test silencer and this had a thickness of $t=1 \mathrm{~mm}$, a hole diameter of $d=3.5 \mathrm{~mm}$ and an area porosity of $\sigma=0.263$. When a mean flow Mach 
number of $M=0.15$ was present the friction velocity was measured as $u_{*}=2.56 \mathrm{~m} / \mathrm{s}$. In Figures 3-6, comparisons are made between experimental measurements and low frequency predictions obtained both with and without a perforate (to omit the perforate from transmission loss calculations one simply sets $\zeta=0$ in the coefficients of equation (27)).

It is evident in Figures 3-6 that generally good agreement exists between measured values for the transmission loss and those calculated using the low-frequency algorithm, both with and without a perforate. Agreement is best at low frequencies, as one would expect, and deteriorates at frequencies above approximately $1 \mathrm{kHz}$. At frequencies above $1.5 \mathrm{kHz}$ caution should be exercised when drawing comparisons between prediction and experiment because the experimental method is known to be inaccurate above $1.5 \mathrm{kHz}$ (see discussion in section 4.1), although data up to $2 \mathrm{kHz}$ is included here since this region is of interest when studying the low-frequency algorithm. For silencer A, good agreement between prediction and experiment is observed up to a frequency of approximately $1 \mathrm{kHz}$ and predictions remain acceptable up to $1.5 \mathrm{kHz}$. Silencer B has a larger outside diameter than silencer A and, unsurprisingly, the low-frequency predictions deviate from measurements at frequencies lower than for silencer A. Silencer $\mathrm{C}$ has the same outside diameter as silencer A but is longer and packed with a different absorbent material. It is evident in Figure 6 that agreement between prediction and experiment is generally similar to that observed for silencer A and this may be expected since the silencers have identical cross-sectional areas.

The low frequency transmission loss predictions generally compare well with experimental measurements however it is evident that predictions obtained with and without a perforate differ, particularly at higher frequencies but also to a lesser extent at lower frequencies. The perforate does not simply behave as if acoustically transparent, as is usually thought for 
screens with relatively high open area porosities $(26.3 \%$ in this case). This effect is thought to be caused by the porous material backing the perforate since this is known to significantly increase the acoustic impedance of the perforate (see reference [17]). After comparison between transmission loss predictions and measurements it is, however, evident that the influence of the perforate may have been overestimated. Overestimation may have been caused by the nature of the perforate impedance measurements performed by Kirby and Cummings [17] since tests performed under idealised laboratory conditions may not accurately represent conditions typically encountered in exhaust silencers. This is manifest in the semi-empirical estimation of the perforate impedance (equation (45)) which assumes a uniformly packed material lies immediately adjacent to the perforate. After the manufacturing process a uniform distribution of absorbent material is unlikely, particularly adjacent to the perforate, and it is thought that in a commercial silencer the overall acoustic impedance of the perforate is significantly reduced in those areas in which the material is not immediately adjacent to the perforate. Therefore, although the transmission loss predictions and measurements presented here do indicate that the acoustic impedance of a perforate, even with a relatively high open area porosity, does significantly influence the overall silencer transmission loss, agreement between prediction and experiment is currently only qualitative. A more accurate representation of the actual perforate impedance in a commercially built dissipative exhaust silencer is probably necessary before improved quantitative agreement is possible. Of course, these observations depend upon comparisons obtained using a low frequency modelling technique. To justify these observation it is necessary to further examine the accuracy of the low-frequency algorithm, at least over the frequency range studied here. A suitable way of doing this is to compare the low frequency algorithm with predictions obtained using a more comprehensive modelling technique. 
To provide further insight into the accuracy of the low frequency algorithm, the method is compared here against a "benchmark" theoretical model. The benchmark predictions are chosen to be those provided by the finite element method described by Peat and Rathi [1] in the belief that this method provides a complete representation of the problem as higher order modes are included implicitly in the transmission loss predictions. Peat and Rathi did, however, omit the effect of a perforate screen so comparisons between finite element predictions and the low-frequency algorithm are possible here only after omitting the perforate screen from the low-frequency computations. For each silencer, eight noded quadrilateral elements were used to construct a finite element mesh which contained 393 nodes for silencer A, 413 nodes for silencer B and 441 nodes for silencer C. In Figures 7-12, the finite element transmission loss predictions are compared with the low-frequency algorithm for silencers A-C, after omitting a perforate and with $M=0$ and 0.15 . Also included in Figures 7 and 8 are transmission loss predictions obtained using the transfer matrix approach of Peat [12] since these serve to illustrate the additional benefit gained from extending the low argument approximations of the Bessel and Neumann functions to higher orders.

It is evident from Figures 7-12 that the general trends observed when comparing the lowfrequency algorithm with experimental transmission loss measurements are apparent also after comparison with the finite element predictions. For instance, the low-frequency algorithm provides good correlation with finite element predictions up to approximately 1500 $\mathrm{Hz}$ for silencers $\mathrm{A}$ and $\mathrm{C}$, and $800 \mathrm{~Hz}$ for silencer B. Moreover, at low frequencies the low frequency algorithm provides an almost exact correlation with finite element predictions for each silencer studied, deviating only slightly when mean flow is present. The benefits of retaining a greater number of terms when expanding the Bessel and Neumann functions in the 
governing eigenequation, and also in the matching conditions, are shown for silencer $\mathrm{A}$ in Figures 7 and 8 . It is clear that the method of Peat [12] is adequate only at very low frequencies, and the method is not sufficiently accurate to be used for studying larger dissipative silencers. Figures 7 and 8 successfully illustrate the motivation behind the new formulation since here it is obvious here that the method of Peat is unsuitable for silencers of a size commonly found in automobiles. Moreover, the new approach now agrees well with the finite element predictions and in some cases replicates the numerical technique up to frequencies approaching $2 \mathrm{kHz}$, although this does depend upon the diameter of the silencer. Nevertheless the low-frequency algorithm appears capable of being utilised as very fast (predictions are instantaneous) and relatively accurate iterative design tool for circular silencers and may successfully replace more computationally onerous techniques provided one is interested in the low to medium frequency range only (often the case in commercial silencer design).

To utilise successfully the low-frequency algorithm for design purposes it is important to be aware of its limitations. The accuracy of the method depends both on the size of the silencer and on the type of porous material contained within the silencer chamber. After examining a number of different silencer configurations, the following relationship is proposed here for defining an approximate upper frequency limit $\left(f_{\max }\right)$, above which the low frequency algorithm should not be used.

$$
f_{\max }=0.43 \frac{c_{0}}{r_{2}}\left\{1-0.06 \frac{S_{2}}{S_{1}}\right\} .
$$


This limit corresponds to a frequency at which the difference between transmission loss predictions obtained by using the low-frequency algorithm and those found using the finite element method is approximately $5 \mathrm{~dB}$. This expression depends upon assuming that the dimensionless bulk compressibility of the porous material tends, in the high frequency limit, towards a value of $\left(\gamma_{0}+\mathrm{i} 0\right)$. This assumption effectively suppresses the influence of the porous material and therefore provides only very a approximate relationship for $f_{\max }$, nevertheless, for the silencers studied here values for $f_{\max }$ appear to be reasonable. For example, for silencers $\mathrm{A}$ and $\mathrm{C}$ equation (51) fixes an upper frequency limit of $f_{\max }=1560 \mathrm{~Hz}$ and for silencer B $f_{\max }=880 \mathrm{~Hz}$.

\section{CONCLUSIONS}

For frequencies below $f_{\max }$ (see equation (51)) the low-frequency algorithm provides good correlation both with experimental measurement and with more sophisticated modelling techniques such as the finite element method. The low-frequency algorithm is suitable for use as a fast and relatively accurate tool for designing circular dissipative silencers and may usefully avoid computationally expensive techniques provided one is not studying relatively large silencers and/or medium to high frequencies. Furthermore, the predictions presented here show that, even for high open area porosities, the perforate screen has a significant effect on dissipative silencer performance, although further work is required to provide more reliable impedance data for commercially produced silencers. 


\section{REFERENCES}

1. K.S. Peat and K.L. Rathi 1995 Journal of Sound and Vibration 184, 529-545. A finite element analysis of the convected acoustic wave motion in dissipative silencers.

2. R.J. Astley and A. Cummings 1987 Journal of Sound and Vibration 116, 239-263. A finite element scheme for attenuation in ducts lined with porous material: comparison with experiment.

3. A.F. Seybert, R.A. Seman and M.D. Lattuca 1998 Transactions of the ASME 120, 976981. Boundary element prediction of sound propagation in ducts containing bulk absorbing materials.

4. R. Kirby 1996 PhD Thesis, University of Hull. The acoustic modelling of dissipative elements in automotive exhausts.

5. S.-H. Ko 1975 Journal of Sound and Vibration 39, 471-487. Theoretical analyses of sound attenuation in acoustically lined flow ducts separated by porous splitters (rectangular, annular and circular ducts).

6. B. Nilsson and O. Brander 1980 IMA Journal of Applied Mathematics 26, 269-298. The propagation of sound in cylindrical ducts with mean flow and bulk reacting lining. I. Modes in and infinite duct.

7. A. Cummings and I.-J. Chang 1987 Acustica 64, 170-178. Internal mean flow effects on the characteristics of bulk-reacting liners in circular ducts.

8. W. Frommhold and F.P. Mechel 1990 Journal of Sound and Vibration 141, 103-125. Simplified methods to calculate the attenuation of silencers.

9. F.P. Mechel 1998 Acustica 84, 201-222. Modal solutions in circular and annular ducts with locally or bulk reacting lining. 
10. B. Nilsson and O. Brander 1980 IMA Journal of Applied Mathematics 27, 263-289. The propagation of sound in cylindrical ducts with mean flow and bulk reacting lining. IV. Several interacting discontinuities.

11. A. Cummings and I.-J. Chang 1988 Journal of Sound and Vibration 127, 1-17. Sound attenuation of a finite length dissipative flow duct silencer with internal mean flow in the absorbent.

12. K.S. Peat 1991 Journal of Sound and Vibration 146, 353-360. A transfer matrix for an absorption silencer element.

13. M. Abramowitz and I.A. Stegun 1965 Handbook of Mathematical Functions. New York: Dover.

14. M.E. Delany and E.N. Bazley 1970 Applied Acoustics 3, 105-116. Acoustical properties of fibrous materials.

15. K.S. Peat 1995 Proceedings of Euro-Noise 95, 791-796. LAMPS software for the acoustic analysis of silencers.

16. R. Kirby and A. Cummings 1999 Applied Acoustics 56, 101-125. Prediction of the bulk acoustic properties of fibrous materials at low frequencies.

17. R. Kirby and A. Cummings 1998 Journal of Sound and Vibration 217, 619-636. The impedance of perforated plates subjected to grazing gas flow and backed by porous media.

18. K.S. Peat 1990 Proceedings of Inter-Noise 90 1, 579-582. A numerical decoupling analysis of absorption silencer elements. 


\section{APPENDIX A: COEFFICIENTS OF EIGENEQUATION}

$$
\begin{aligned}
& H_{00}=\frac{S_{1}}{S_{2}} \frac{\rho(\omega)}{\rho_{0}}-\tilde{\Gamma}^{2}\left(1+\frac{\mathrm{i} \zeta k_{0} r_{1}}{2}\right)-\frac{k_{0}^{2} r_{1}^{2}}{8}\left\{\frac{S_{1}}{S_{2}} \frac{\rho(\omega)}{\rho_{0}}-\tilde{\Gamma}^{2}\left(2+\frac{\mathrm{i} \zeta k_{0} r_{1}}{2}\right)\right\} \\
& +\frac{k_{0}^{2} r_{2}^{2} \tilde{\Gamma}^{2}}{8}\left\{2 R_{1} \frac{S_{1}}{S_{2}} \frac{\rho(\omega)}{\rho_{0}}+\tilde{\Gamma}^{2} R_{2}\left(1+\frac{\mathrm{i} \zeta k_{0} r_{1}}{2}\right)\right\}-\frac{k_{0}^{4} r_{1}^{2} r_{2}^{2} \tilde{\Gamma}^{2}}{64}\left\{2 R_{1} \frac{S_{1}}{S_{2}} \frac{\rho(\omega)}{\rho_{0}}+\tilde{\Gamma}^{2} R_{2}\left(2+\frac{\mathrm{i} \zeta k_{0} r_{1}}{2}\right)\right\} \\
& +\frac{k_{0}^{4} r_{2}^{4} \tilde{\Gamma}^{4}}{192}\left\{3 R_{3} \frac{S_{1}}{S_{2}} \frac{\rho(\omega)}{\rho_{0}}+\tilde{\Gamma}^{2} R_{4}\left(1+\frac{i \zeta k_{0} r_{1}}{2}\right)\right\} \\
& H_{11}=\frac{S_{1}}{S_{2}} \frac{\rho(\omega)}{\rho_{0}}-\tilde{\Gamma}^{2}\left(1+\frac{\mathrm{i} \zeta k_{0} r_{1}}{2}\right)-\frac{k_{0}^{2} r_{1}^{2}}{4}\left\{\frac{S_{1}}{S_{2}} \frac{\rho(\omega)}{\rho_{0}}-\tilde{\Gamma}^{2}\left(2+\frac{\mathrm{i} \zeta k_{0} r_{1}}{2}\right)\right\} \\
& +\frac{k_{0}^{2} r_{2}^{2} \tilde{\Gamma}^{2}}{8}\left\{2 R_{1} \frac{S_{1}}{S_{2}} \frac{\rho(\omega)}{\rho_{0}}+\tilde{\Gamma}^{2} R_{2}\left(1+\frac{\mathrm{i} \zeta k_{0} r_{1}}{2}\right)\right\}-\frac{k_{0}^{4} r_{1}^{2} r_{2}^{2} \tilde{\Gamma}^{2}}{32}\left\{2 R_{1} \frac{S_{1}}{S_{2}} \frac{\rho(\omega)}{\rho_{0}}+\tilde{\Gamma}^{2} R_{2}\left(2+\frac{\mathrm{i} \zeta k_{0} r_{1}}{2}\right)\right\} \\
& +\frac{k_{0}^{4} r_{2}^{4} \tilde{\Gamma}^{4}}{192}\left\{3 R_{3} \frac{S_{1}}{S_{2}} \frac{\rho(\omega)}{\rho_{0}}+\tilde{\Gamma}^{2} R_{4}\left(1+\frac{\mathrm{i} \zeta k_{0} r_{1}}{2}\right)\right\} \\
& H_{20}=1+\frac{S_{1}}{S_{2}} \frac{\rho(\omega)}{\rho_{0}}+\frac{\mathrm{i} \zeta k_{0} r_{1}}{2}\left(1-\tilde{\Gamma}^{2}\right)-\frac{k_{0}^{2} r_{1}^{2}}{4}\left\{\frac{S_{1}}{S_{2}} \frac{\rho(\omega)}{\rho_{0}}+1-\tilde{\Gamma}^{2}\left(1+\frac{\mathrm{i} \zeta k_{0} r_{1}}{2}\right)\right\} \\
& -\frac{k_{0}^{2} r_{2}^{2}}{8}\left\{2 R_{1} \frac{S_{1}}{S_{2}} \frac{\rho(\omega)}{\rho_{0}}\left(1-\tilde{\Gamma}^{2}\right)+2 R_{2} \tilde{\Gamma}^{2}+\frac{\mathrm{i} \zeta k_{0} r_{1}}{2} R_{2} \tilde{\Gamma}^{2}\left(2-\tilde{\Gamma}^{2}\right)\right\} \\
& -\frac{k_{0}^{4} r_{1}^{2} r_{2}^{2} \tilde{\Gamma}^{2}}{32}\left\{2 R_{1} \frac{S_{1}}{S_{2}} \frac{\rho(\omega)}{\rho_{0}}-R_{2}+R_{2} \tilde{\Gamma}^{2}\left(1+\frac{\mathrm{i} \zeta k_{0} r_{1}}{2}\right)\right\} \\
& -\frac{k_{0}^{4} r_{2}^{4} \tilde{\Gamma}^{2}}{192}\left\{3 R_{3} \frac{S_{1}}{S_{2}} \frac{\rho(\omega)}{\rho_{0}}\left(2-\tilde{\Gamma}^{2}\right)+3 R_{4} \tilde{\Gamma}^{2}+\frac{\mathrm{i} \zeta k_{0} r_{1}}{2} R_{4} \tilde{\Gamma}^{2}\left(3-\tilde{\Gamma}^{2}\right)\right\}
\end{aligned}
$$




$$
\begin{aligned}
& H_{22}=\frac{S_{1}}{S_{2}} \frac{\rho(\omega)}{\rho_{0}}-\tilde{\Gamma}^{2}\left(1+\frac{\mathrm{i} \zeta k_{0} r_{1}}{2}\right)-\frac{k_{0}^{2} r_{1}^{2}}{8}\left\{6 \frac{S_{1}}{S_{2}} \frac{\rho(\omega)}{\rho_{0}}-\tilde{\Gamma}^{2}\left(11+3 \mathrm{i} \zeta k_{0} r_{1}\right)\right\} \\
& +\frac{k_{0}^{2} r_{2}^{2} \tilde{\Gamma}^{2}}{8}\left\{2 R_{1} \frac{S_{1}}{S_{2}} \frac{\rho(\omega)}{\rho_{0}}+\tilde{\Gamma}^{2} R_{2}\left(1+\frac{\mathrm{i} \zeta k_{0} r_{1}}{2}\right)\right\}-\frac{3 k_{0}^{4} r_{1}^{2} r_{2}^{2} \tilde{\Gamma}^{2}}{32}\left\{2 R_{1} \frac{S_{1}}{S_{2}} \frac{\rho(\omega)}{\rho_{0}}+\tilde{\Gamma}^{2} R_{2}\left(2+\frac{\mathrm{i} \zeta k_{0} r_{1}}{2}\right)\right\} \\
& +\frac{k_{0}^{4} r_{2}^{4} \tilde{\Gamma}^{4}}{192}\left\{3 R_{3} \frac{S_{1}}{S_{2}} \frac{\rho(\omega)}{\rho_{0}}+\tilde{\Gamma}^{2} R_{4}\left(1+\frac{\mathrm{i} \zeta k_{0} r_{1}}{2}\right)\right\} \\
& H_{31}=2\left(1+\frac{\mathrm{i} \zeta k_{0} r_{1}}{2}\right)-\frac{k_{0}^{2} r_{1}^{2}}{2}\left\{\frac{S_{1}}{S_{2}} \frac{\rho(\omega)}{\rho_{0}}+2-\tilde{\Gamma}^{2}\left(1+\frac{\mathrm{i} \zeta k_{0} r_{1}}{2}\right)\right\} \\
& -\frac{k_{0}^{2} r_{2}^{2}}{2}\left\{R_{1} \frac{S_{1}}{S_{2}} \frac{\rho(\omega)}{\rho_{0}}+\tilde{\Gamma}^{2} R_{2}\left(1+\frac{\mathrm{i} \zeta k_{0} r_{1}}{2}\right)\right\} \\
& -\frac{k_{0}^{4} r_{1}^{2} r_{2}^{2} \tilde{\Gamma}^{2}}{16}\left\{2 R_{1} \frac{S_{1}}{S_{2}} \frac{\rho(\omega)}{\rho_{0}}-2 R_{2}+R_{2} \tilde{\Gamma}^{2}\left(1+\frac{\mathrm{i} \zeta k_{0} r_{1}}{2}\right)\right\} \\
& -\frac{k_{0}^{4} r_{2}^{4} \tilde{\Gamma}^{2}}{32}\left\{2 R_{3} \frac{S_{1}}{S_{2}} \frac{\rho(\omega)}{\rho_{0}}+\tilde{\Gamma}^{2} R_{4}\left(1+\frac{\mathrm{i} \zeta k_{0} r_{1}}{2}\right)\right\} \\
& H_{40}=\frac{\mathrm{i} \zeta k_{0} r_{1}}{2}-\frac{k_{0}^{2} r_{1}^{2}}{8}\left\{\frac{S_{1}}{S_{2}} \frac{\rho(\omega)}{\rho_{0}}+2-\tilde{\Gamma}^{2} \frac{\mathrm{i} \zeta k_{0} r_{1}}{2}\right\} \\
& -\frac{k_{0}^{2} r_{2}^{2}}{8}\left\{2 R_{1} \frac{S_{1}}{S_{2}} \frac{\rho(\omega)}{\rho_{0}}-R_{2}\left(1-\mathrm{i} \zeta k_{0} r_{1} \tilde{\Gamma}^{2}\right)\right\} \\
& -\frac{k_{0}^{4} r_{1}^{2} r_{2}^{2} \tilde{\Gamma}^{2}}{64}\left\{2 R_{1} \frac{S_{1}}{S_{2}} \frac{\rho(\omega)}{\rho_{0}}-R_{2}\left(2-\tilde{\Gamma}^{2} \frac{\mathrm{i} \zeta k_{0} r_{1}}{2}\right)\right\} \\
& -\frac{k_{0}^{4} r_{2}^{4} \tilde{\Gamma}^{2}}{192}\left\{6 R_{3} \frac{S_{1}}{S_{2}} \frac{\rho(\omega)}{\rho_{0}}-R_{4}\left(2-3 \tilde{\Gamma}^{2} \frac{\mathrm{i} \zeta k_{0} r_{1}}{2}\right)\right\}
\end{aligned}
$$




$$
\begin{aligned}
& H_{42}=1+\frac{\mathrm{i} \zeta k_{0} r_{1}}{2}-\frac{k_{0}^{2} r_{1}^{2}}{8}\left\{2 \frac{S_{1}}{S_{2}} \frac{\rho(\omega)}{\rho_{0}}+11-2 \tilde{\Gamma}^{2}\left(1+\frac{\mathrm{i} \zeta k_{0} r_{1}}{2}\right)\right\} \\
& -\frac{k_{0}^{2} r_{2}^{2}}{4}\left\{R_{1} \frac{S_{1}}{S_{2}} \frac{\rho(\omega)}{\rho_{0}}+\tilde{\Gamma}^{2} R_{2}\left(1+\frac{\mathrm{i} \zeta k_{0} r_{1}}{2}\right)\right\}- \\
& -\frac{k_{0}^{4} r_{1}^{2} r_{2}^{2} \tilde{\Gamma}^{2}}{32}\left\{2 R_{1} \frac{S_{1}}{S_{2}} \frac{\rho(\omega)}{\rho_{0}}-6 R_{2}+R_{2} \tilde{\Gamma}^{2}\left(1+\frac{\mathrm{i} \zeta k_{0} r_{1}}{2}\right)\right\} \\
& -\frac{k_{0}^{4} r_{2}^{4} \tilde{\Gamma}^{2}}{64}\left\{2 R_{3} \frac{S_{1}}{S_{2}} \frac{\rho(\omega)}{\rho_{0}}+\tilde{\Gamma}^{2} R_{4}\left(1+\frac{\mathrm{i} \zeta k_{0} r_{1}}{2}\right)\right\} \\
& H_{51}=\frac{k_{0}^{2} r_{1}^{2}}{2}-\frac{k_{0}^{2} r_{2}^{2} R_{2}}{4}-\frac{k_{0}^{4} r_{1}^{2} r_{2}^{2} R_{2} \tilde{\Gamma}^{2}}{16}-\frac{k_{0}^{4} r_{2}^{4} R_{4} \tilde{\Gamma}^{2}}{48} \\
& H_{62}=\frac{k_{0}^{2} r_{1}^{2}}{4}-\frac{k_{0}^{2} r_{2}^{2} R_{2}}{8}-\frac{k_{0}^{4} r_{1}^{2} r_{2}^{2} R_{2} \tilde{\Gamma}^{2}}{32}-\frac{k_{0}^{4} r_{2}^{4} R_{4} \tilde{\Gamma}^{2}}{96}
\end{aligned}
$$


TABLES

\begin{tabular}{|c|c|c|c|}
\hline \multicolumn{4}{|c|}{ Table 1. Chamber dimensions of test silencers. } \\
\hline Silencer & Length $L(\mathrm{~mm})$ & Diameter $2 \times r_{2}(\mathrm{~mm})$ & Absorbent \\
\hline A & 315 & 152.4 & E glass \\
\hline B & 330 & 203.2 & E glass \\
\hline C & 450 & 152.4 & A glass \\
\hline
\end{tabular}

\begin{tabular}{|c|c|c|}
\hline \multicolumn{2}{|c|}{ Table 2. Values of material constants. } \\
\hline Constant & E glass & A glass \\
\hline$a_{1}$ & 0.2202 & 0.2251 \\
\hline$a_{2}$ & -0.5850 & -0.5827 \\
\hline$a_{3}$ & 0.2010 & 0.1443 \\
\hline$a_{4}$ & -0.5829 & -0.7088 \\
\hline$a_{5}$ & 0.0954 & 0.0924 \\
\hline$a_{6}$ & -0.6687 & -0.7177 \\
\hline$a_{7}$ & 0.1689 & 0.1457 \\
\hline$a_{8}$ & -0.5707 & -0.5951 \\
\hline$\sigma_{b}(\mathrm{MK}$ rayl $/ \mathrm{m})$ & 30716 & 5976 \\
\hline$\Omega_{2}$ & 0.952 & 0.952 \\
\hline$q_{0}^{2}$ & 5.49 & 3.77 \\
\hline$f_{0}$ & 0.005 & 0.025 \\
\hline
\end{tabular}




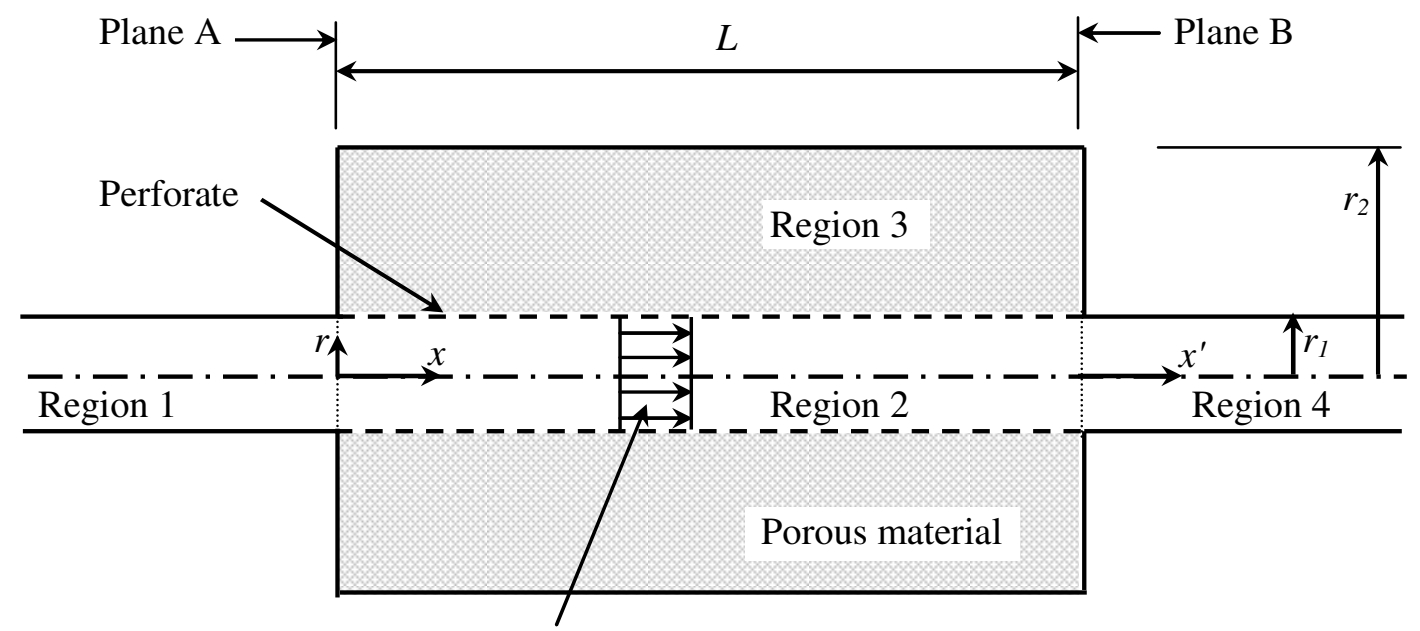

Mean flow mach number $M$

Figure 1. Geometry of dissipative silencer.

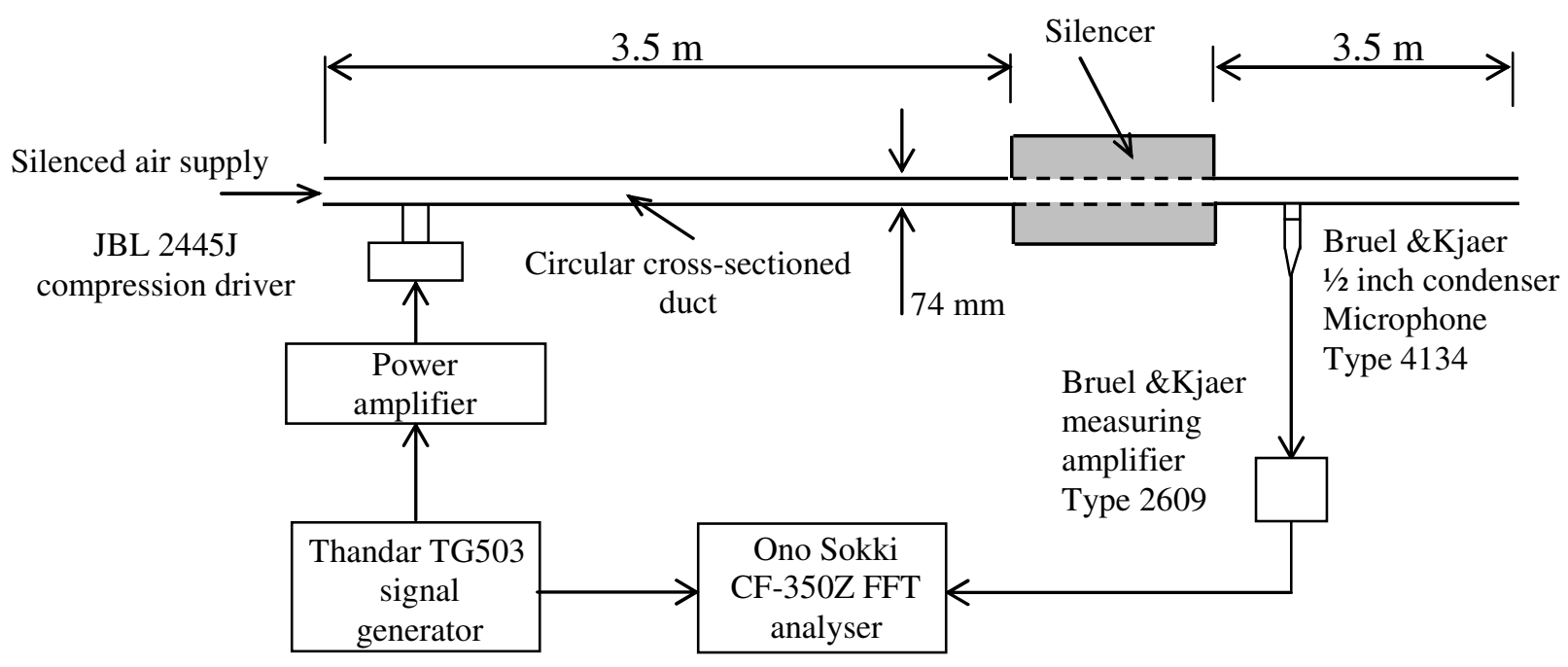

Figure 2. Apparatus for measurement of silencer transmission loss. 


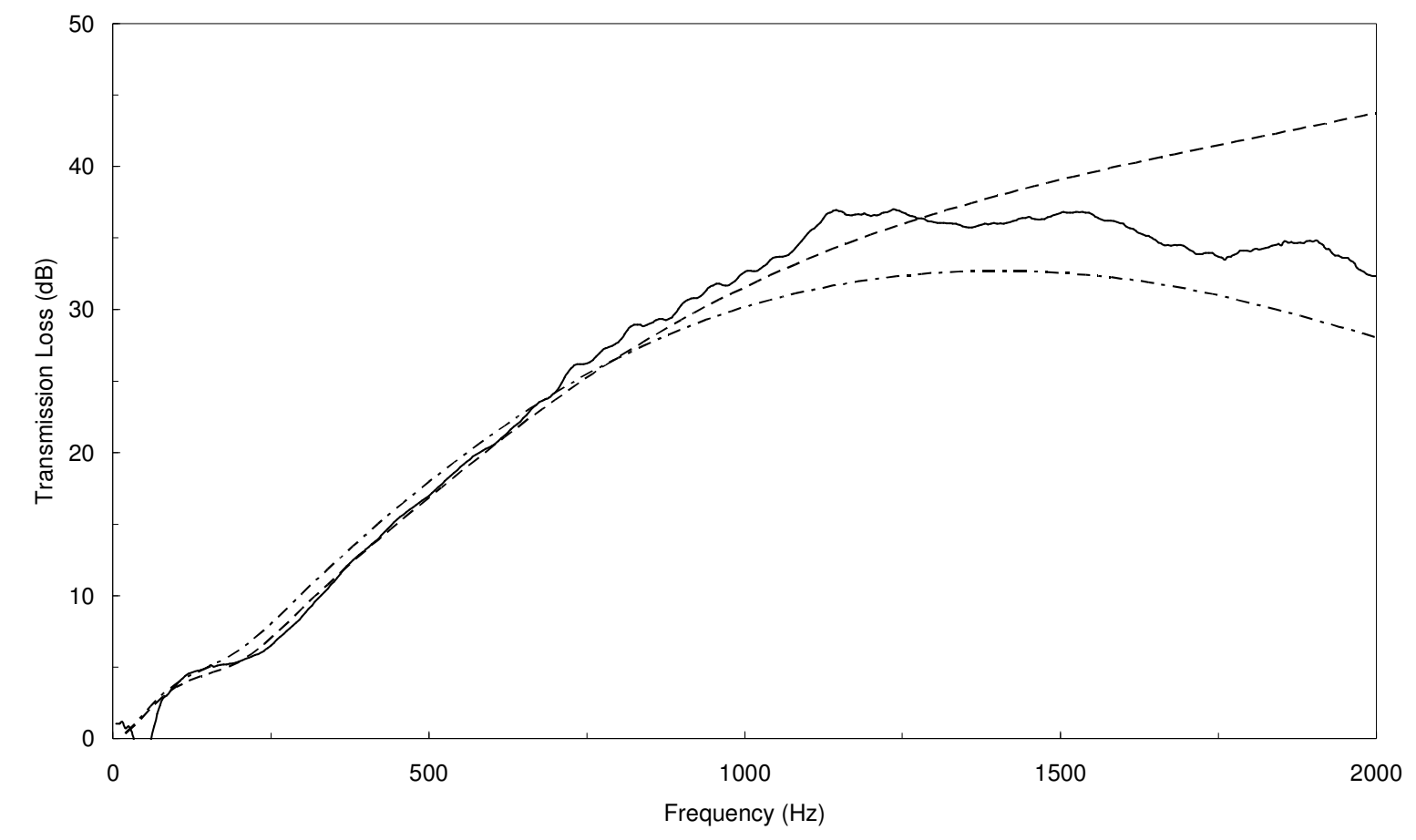

Figure 3. Predicted and measured transmission loss for silencer A, $M=0$ :

experiment; $-\ldots-$, low frequency algorithm (no perforate); $-\cdots-{ }_{-}$, low frequency algorithm (perforate included). 


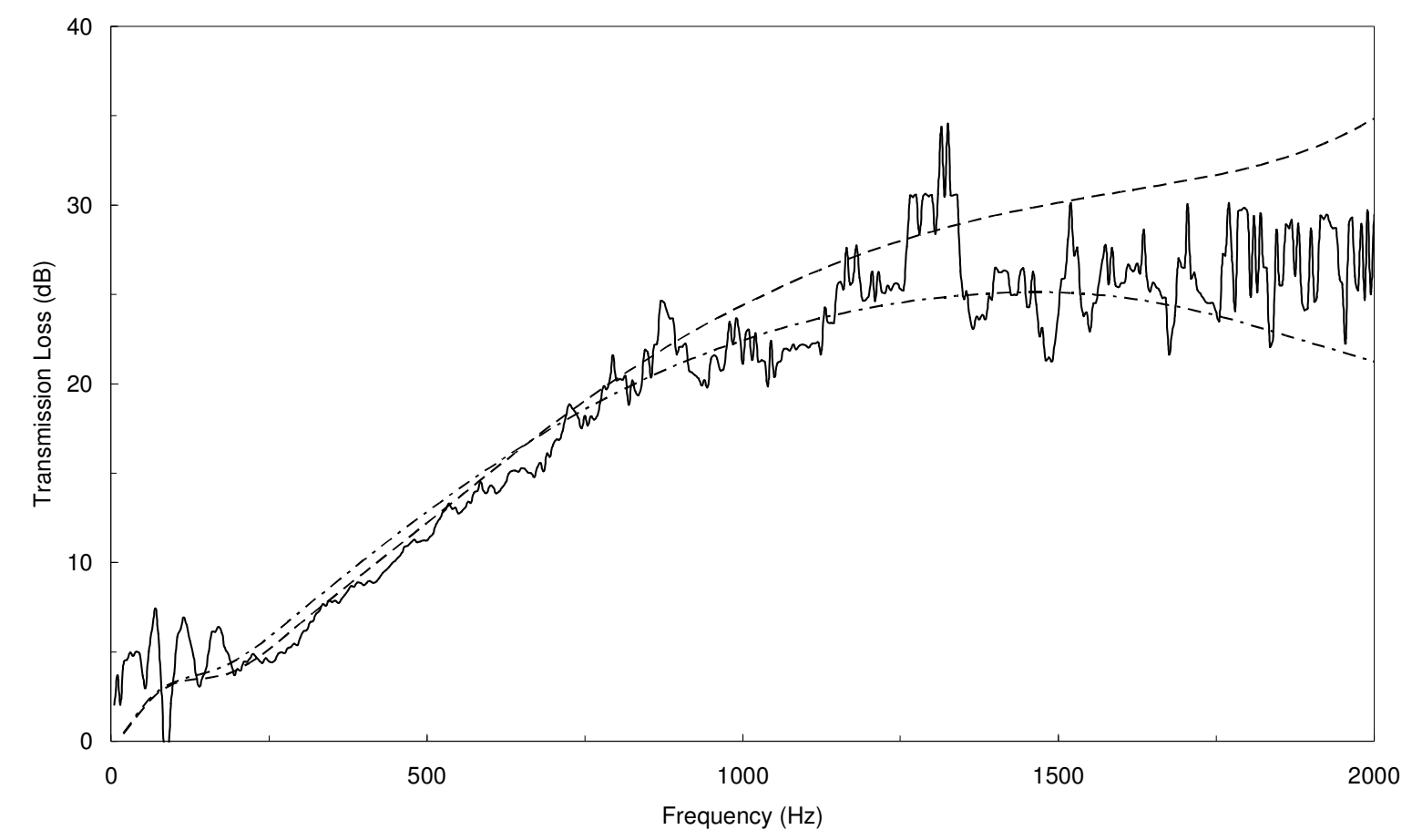

Figure 4. Predicted and measured transmission loss for silencer A, $M=0.15$ :

experiment; $-\ldots-$, low frequency algorithm (no perforate); $-\cdots-\ldots$ frequency algorithm (perforate included). 


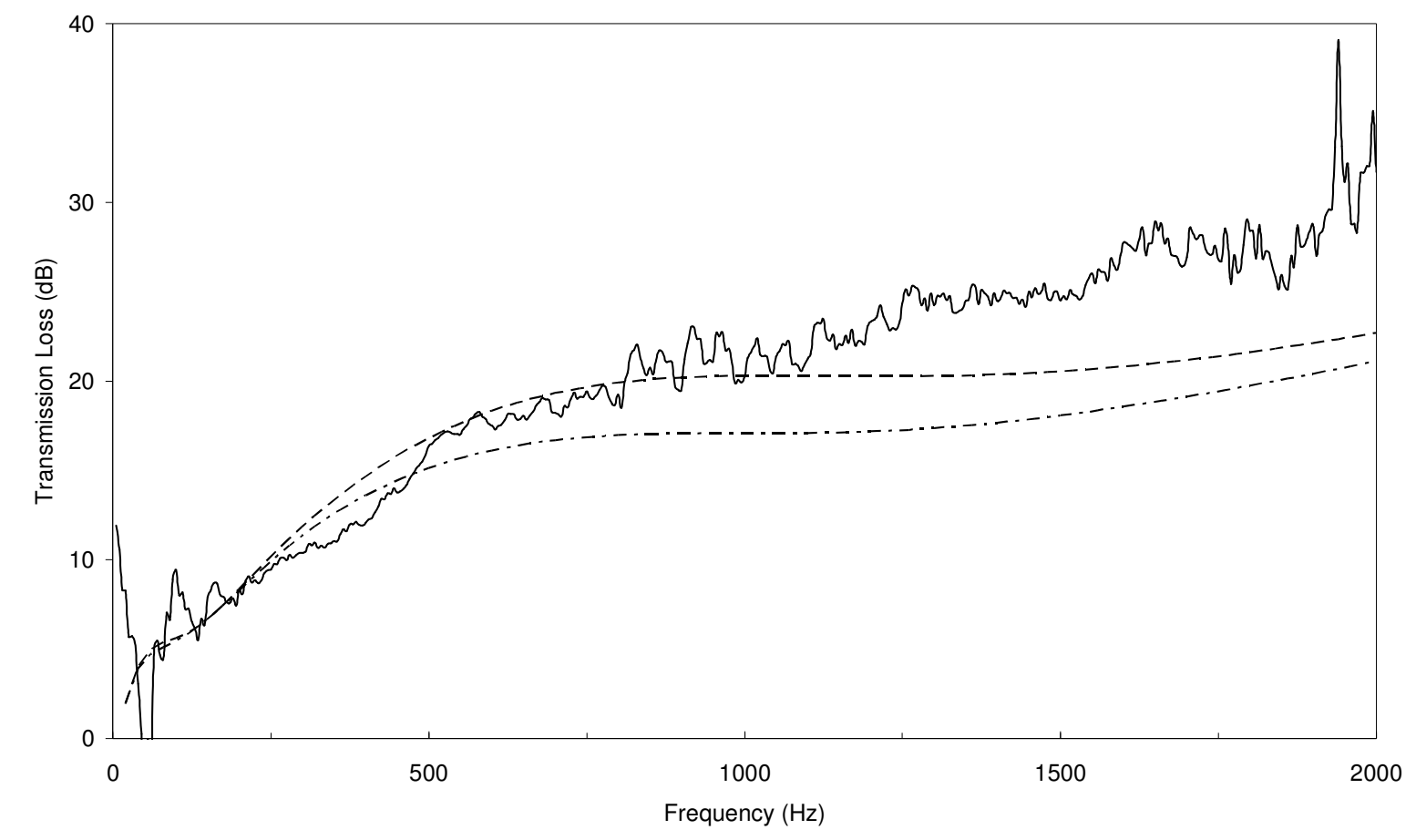

Figure 5. Predicted and measured transmission loss for silencer $\mathrm{B}, M=0.15$ :

experiment; $-\ldots-$, low frequency algorithm (no perforate); $-\cdots-\ldots$ frequency algorithm (perforate included). 


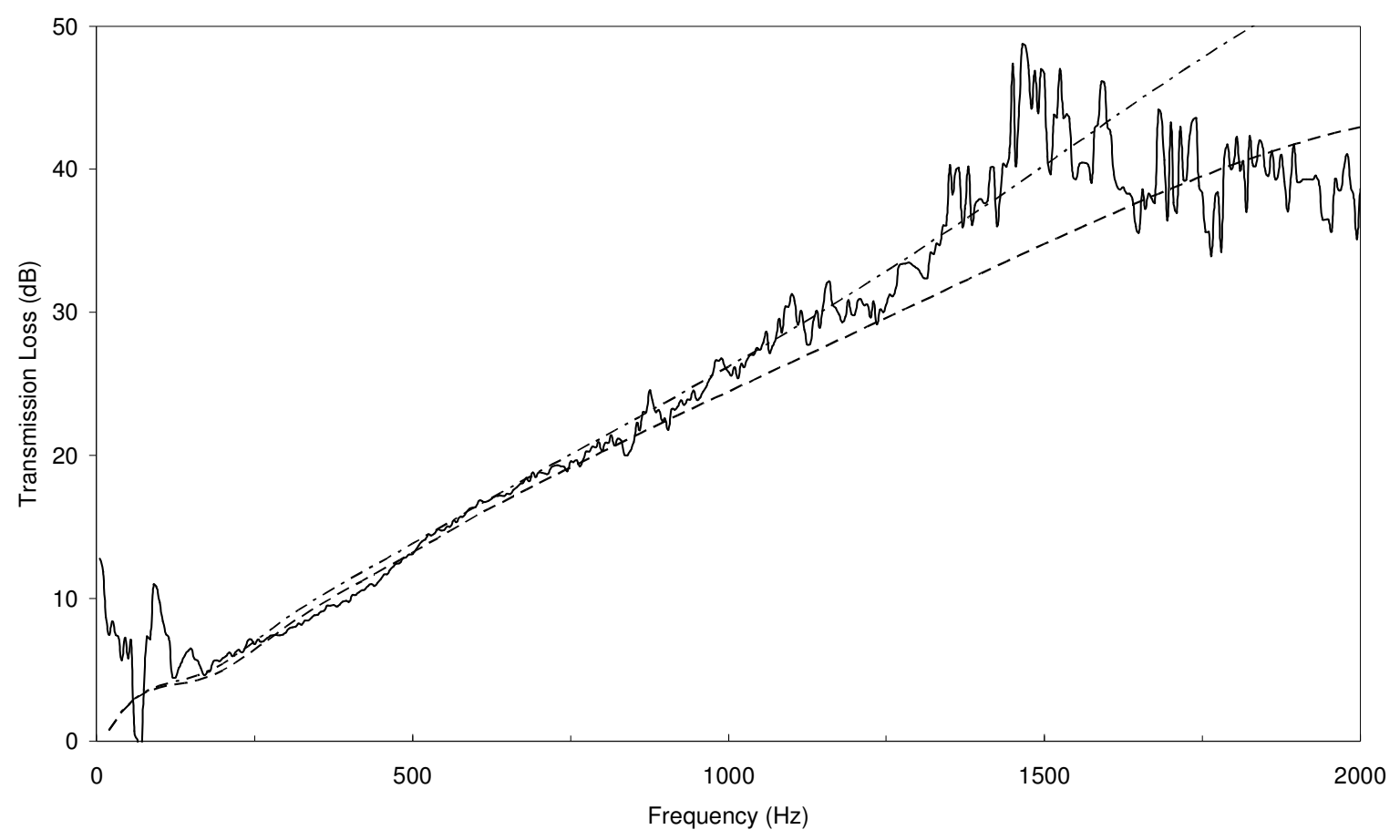

Figure 6. Predicted and measured transmission loss for silencer $\mathrm{C}, M=0.15$ : experiment; $-\ldots-$, low frequency algorithm (no perforate); $-\cdots-{ }_{-}$, low frequency algorithm (perforate included). 


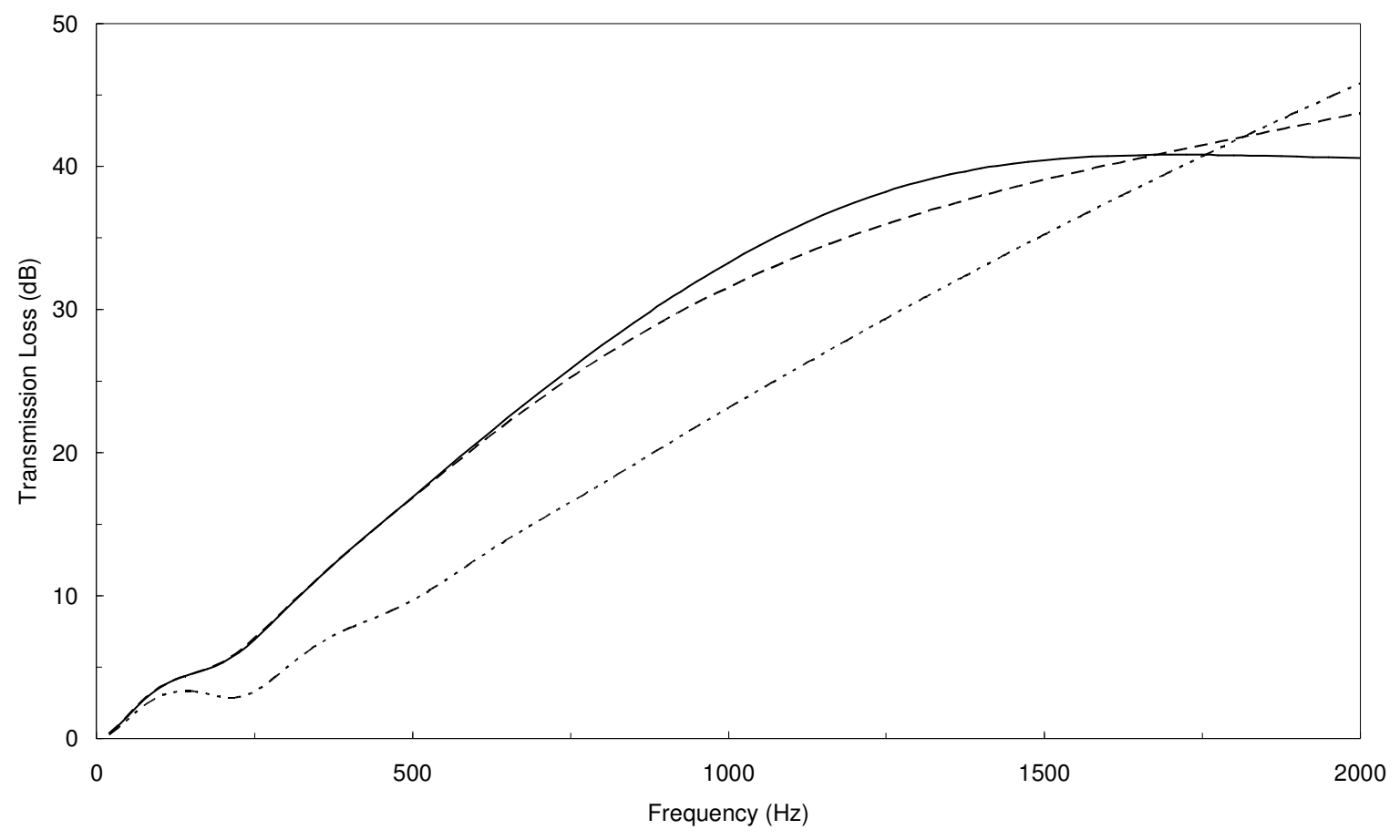

Figure 7. Predicted transmission loss for silencer A (no perforate), $M=0$ : — , finite element method [1]; - — —, low frequency algorithm; $-\cdots---\_$, transfer matrix model [12]. 


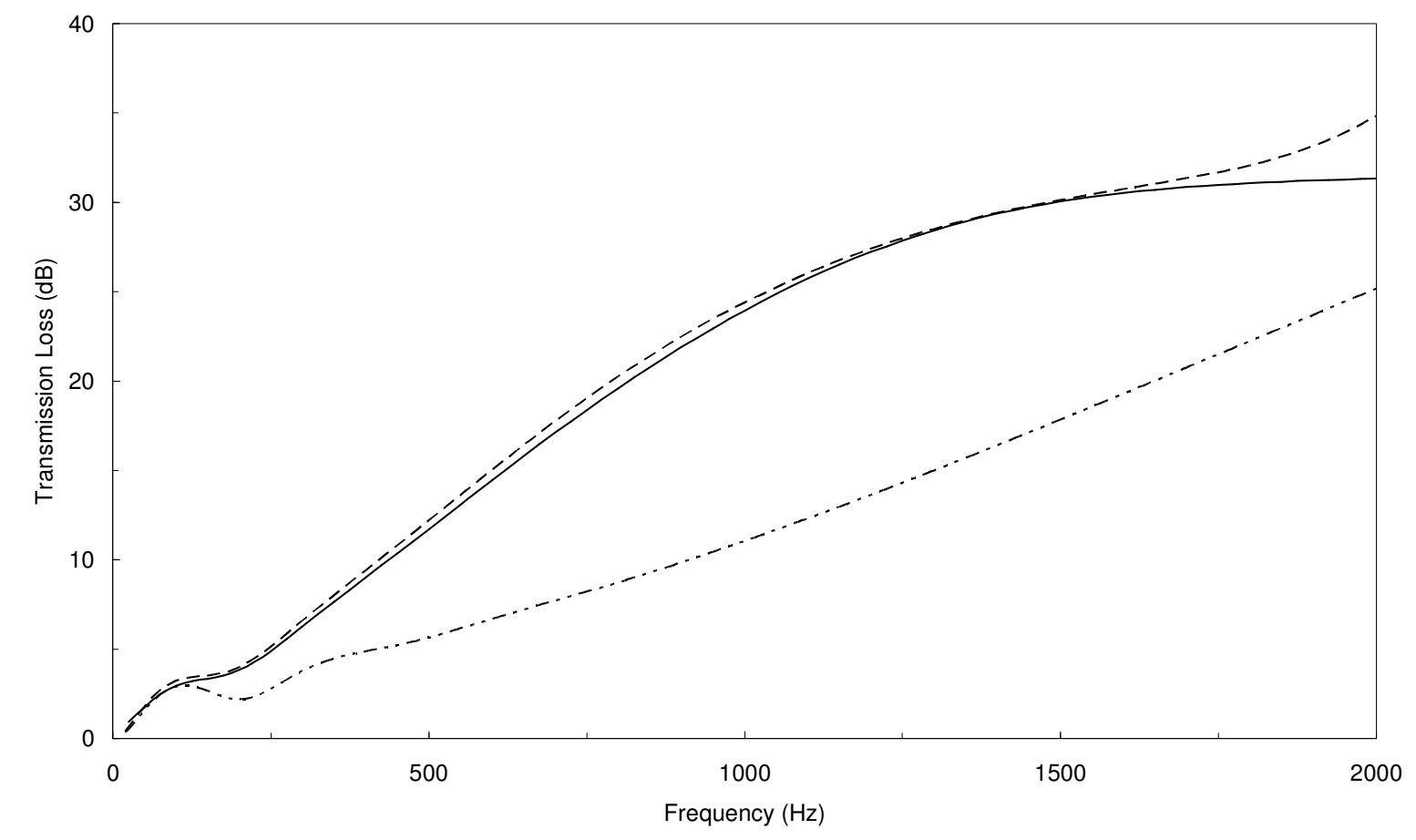

Figure 8. Predicted transmission loss for silencer A (no perforate), $M=0.15:-$, finite element method [1]; - — —, low frequency algorithm; $-\cdots---\_$, transfer matrix model [12]. 


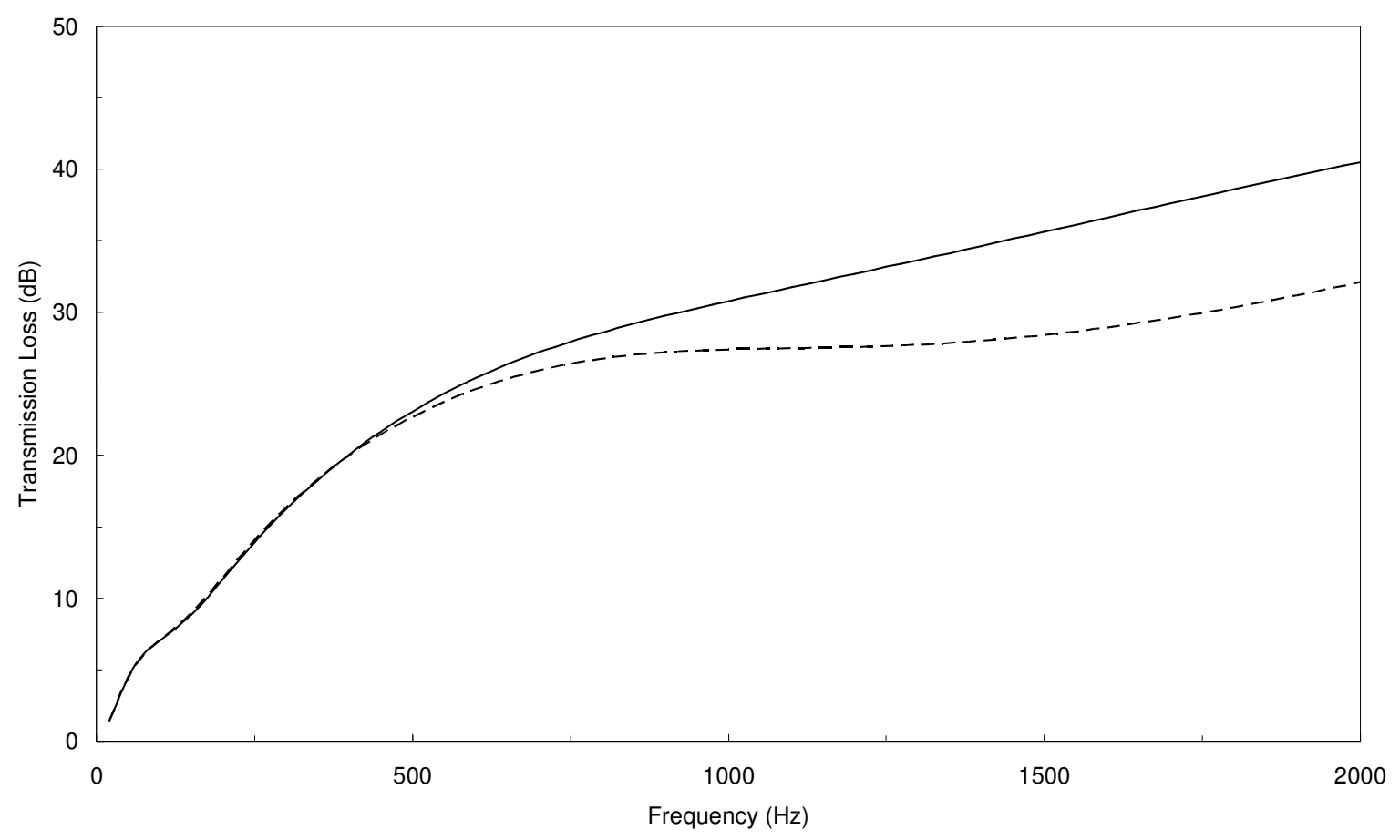

Figure 9. Predicted transmission loss for silencer B (no perforate), $M=0:-$, finite element method $[1] ;-\_-$, low frequency algorithm. 


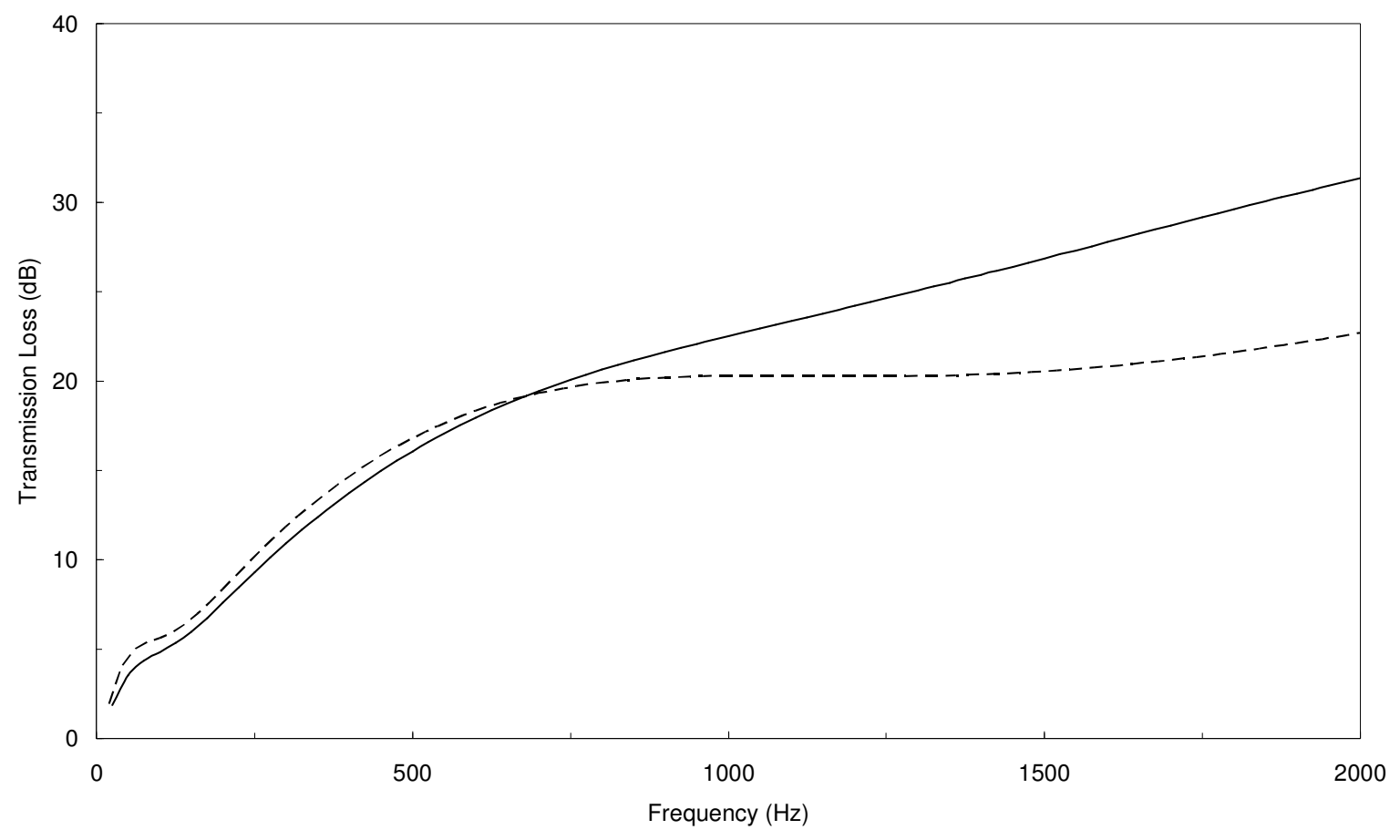

Figure 10. Predicted transmission loss for silencer B (no perforate), $M=0.15$ :

finite element method [1]; - — - low frequency algorithm. 


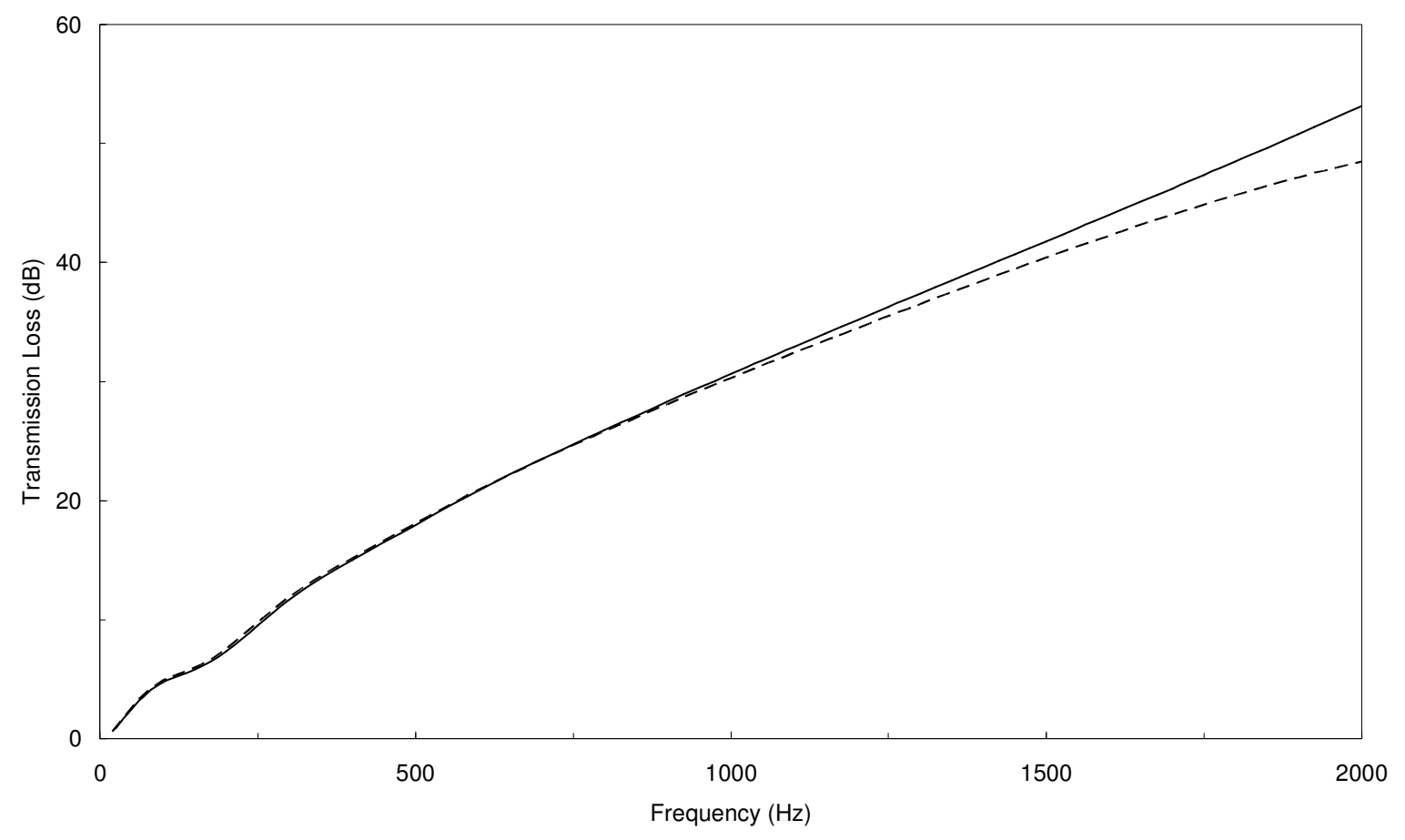

Figure 11. Predicted transmission loss for silencer $\mathrm{C}$ (no perforate), $M=0:-$, finite element method $[1] ;-\_-$, low frequency algorithm. 


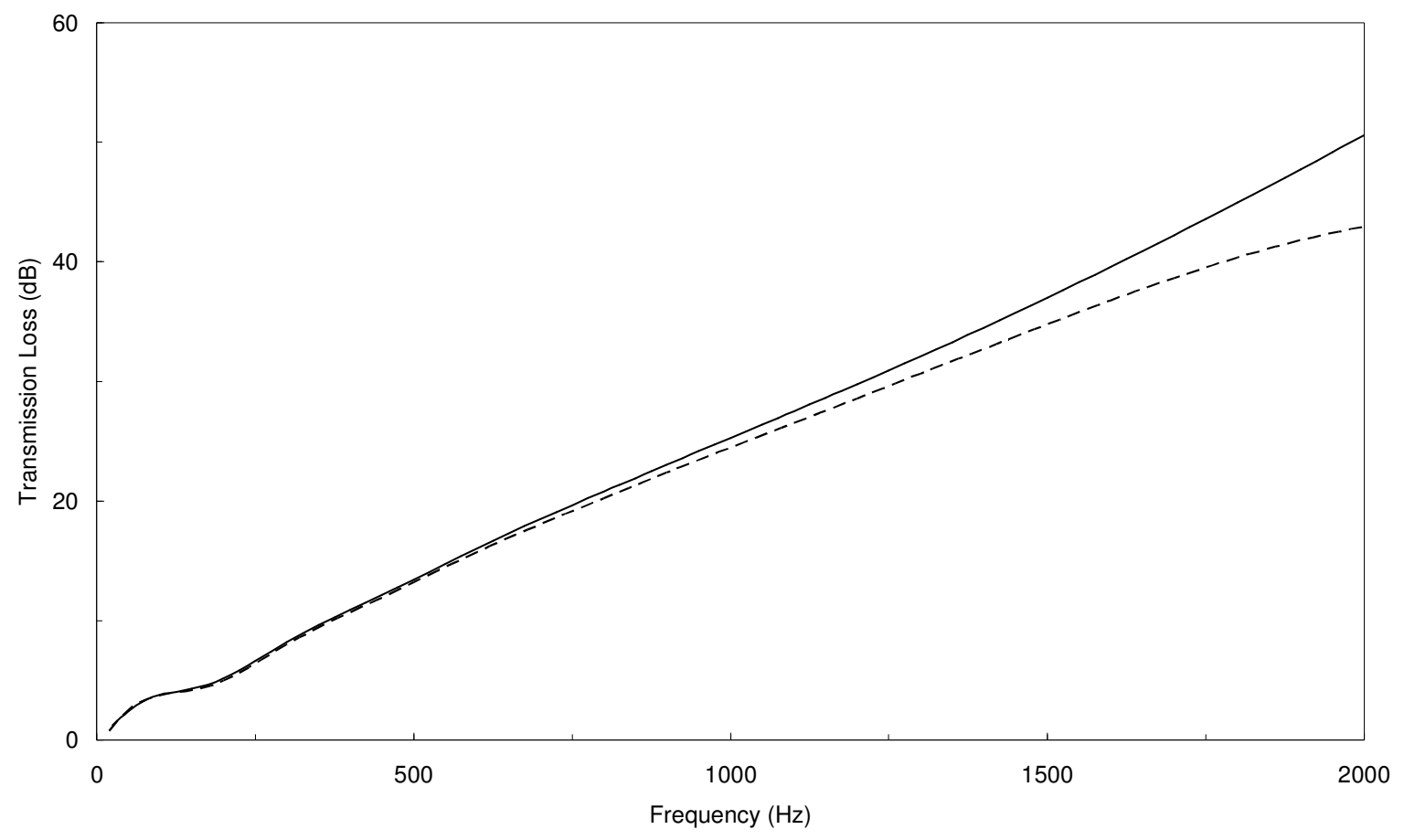

Figure 12. Predicted transmission loss for silencer $\mathrm{C}$ (no perforate), $M=0.15$ :

finite element method [1]; - - , low frequency algorithm. 